\title{
A Comprehensive Review of Electrospinning Block
}

\section{Copolymers}

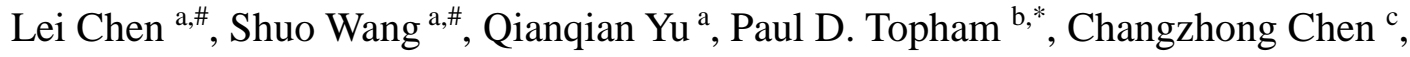
Linge Wang a,*

a South China Advanced Institute for Soft Matter Science and Technology, School of Molecular Science and Engineering, South China University of Technology, Guangzhou 510640, China.

${ }^{\mathrm{b}}$ Aston Institute of Materials Research, Aston University, Birmingham, B4 7ET, UK

${ }^{\mathrm{c}}$ School of Chemistry, Biology and Environmental Engineering, Xiangnan University, Chenzhou 423000, China

\section{Corresponding authors:}

P. D. Topham, Email: p.d.topham@aston.ac.uk, Tel: +44-121-2043413

L. Wang, Email: lingewang@ scut.edu.cn, Tel: +86-20-22237367

\# L. Chen and S. Wang contributed equally

\begin{abstract}
:
Electrospinning provides a versatile and cost-effective route for the generation of continuous nanofibres with high surface area-to-volume ratio from various polymers. In parallel, block copolymers (BCPs) are promising candidates for many diverse applications, where nanoscale operation is exploited, owing to their intrinsic self-assembling behaviour at these length scales. Judicious combination of BCPs (with their ability to make nanosized domains at equilibrium) and electrospinning (with its ability to create nano- and microsized fibres and particles) allows one to create BCPs with high surface area-to-volume ratio to deliver higher efficiency or efficacy in their given application. Here, we give a comprehensive overview of the wide range of reports on $\mathrm{BCP}$ electrospinning with focus placed on the use of molecular design alongside control over specific electrospinning type and post-treatment methodologies
\end{abstract}


to control the properties of the resultant fibrous materials. Particular attention is paid to the applications of these materials, most notably, their use as biomaterials, separation membranes, sensors, and optoelectronic materials.

\section{Key Words:}

Electrospinning; Block copolymers; Nanofibres; Microphase separation;

Self-Assembly; Properties; Applications

\section{Glossary}

\begin{tabular}{|c|c|c|c|}
\hline \multicolumn{4}{|c|}{ Abbreviation } \\
\hline $\mathrm{ABCs}$ & amphiphilic block copolymers & HFIP & hexafluoroisopropanol \\
\hline ADSCs & adipose derived stem cells & IEC & ion-exchange capacity \\
\hline ATRP & Atom Transfer Radical Polymerization & $\mathrm{PF}$ & poly[2,7-(9,9-dihexylfluorene)] \\
\hline A-F127 & acylated poloxamer F127 & PFDA & polyheptadecafluorodecylacrylate \\
\hline$B$ & block & PGA & poly(glycolic acid) \\
\hline BCPs & block copolymers & PHB & poly(hydroxybutyrate) \\
\hline BEI & Backscattered Electron Imaging & PLCL & $\operatorname{poly}(\varepsilon$-caprolactone-co-lactide) \\
\hline BSA & bovine serum albumin & PLGA & poly(lactide-co-glycolide) \\
\hline CNT & carbon nanotubes & PLLA & poly(L-lactide) \\
\hline DSC & Differential Scanning Calorimetry & PMAA & poly(methacrylic acid) \\
\hline DCM & dichlormethane & PMMA & poly(methyl methacrylate) \\
\hline DMF & dimethylfumarate & PMPC & $\begin{array}{l}\text { poly[(2-methacryloyloxy)ethyl } \\
\text { phosphorylcholine }]\end{array}$ \\
\hline DMSO & dimethylsulfoxide & PMPEOMA & $\begin{array}{l}\text { poly[methoxy poly(ethylene oxide) } \\
\text { methacrylate] }\end{array}$ \\
\hline $\mathrm{ECM}$ & extracellular matrix & PNIPAM & poly( $N$-isopropylacrylamide) \\
\hline EMS & $\begin{array}{l}\text { perfluorooctylethylene oxymethyl } \\
\text { styrene }\end{array}$ & PNMA & $\operatorname{poly}(N$-methylolacrylamide $)$ \\
\hline FDA & Food and Drug Administration & POEOMA & $\begin{array}{l}\text { poly[oligo(ethylene oxide) } \\
\text { methacrylate] }\end{array}$ \\
\hline FMA & perfluorooctylethylmethacrylate & PPO & poly(propylene oxide) \\
\hline FS & pentafluorostyrene & PPy & poly(1-pyrenemethylmethacrylate) \\
\hline FSF & $\begin{array}{l}\text { 2,3,5,6-tetrafluoro-4-(3,3,4,4,5,5,6,6,7,7, } \\
8,8,9,9,10,10,10 \\
\text { heptadecafluorodecaoxy) styrene }\end{array}$ & PS & polystyrene \\
\hline
\end{tabular}




\begin{tabular}{|c|c|c|c|}
\hline IEFs & ion-exchange fibres & PSBMA & poly(sulfobetaine methacrylate) \\
\hline MF & microfiltration & PTHF & poly(tetrahydrofuran) \\
\hline MOS & metal oxide semiconductor & PU & polyurethane \\
\hline NPs & nanoparticles & PVP & poly(vinyl pyrrolidone) \\
\hline OCA & Oil Contact Angle & P2VP & poly(2-vinylpyridine) \\
\hline OPV & oligophenylenevinylene & P4VP & poly(4-vinyl pyridine) \\
\hline OT & oligothiophene & $\mathrm{RhB}$ & rhodamine B \\
\hline PAN & polyacrylonitrile & SBS & $\begin{array}{l}\text { polystyrene- } b \text {-polybutadiene- } b \text {-pol } \\
\text { ystyrene }\end{array}$ \\
\hline PBA & poly(butyl acrylate) & SEBS & $\begin{array}{l}\text { polystyrene- } b \text {-poly(ethylene-co-bu } \\
\text { tylene)- } b \text {-polystyrene }\end{array}$ \\
\hline PBT & poly(butylene terephthalate) & SELPs & silk-elastin-like protein polymers \\
\hline PCEC & $\begin{array}{l}\text { poly( } \varepsilon \text {-caprolactone)- } b \text {-poly(ethylene } \\
\text { oxide)- } b \text {-poly( } \varepsilon \text {-caprolactone) }\end{array}$ & SEM & Scanning Electron Microscopy \\
\hline PCL & poly( $\varepsilon$-caprolactone $)$ & SF & silk fibroin \\
\hline PDEA & poly[2-(diethylamino)ethyl methacrylate] & SI & poly(styrene- $b$-isoprene) \\
\hline PDLA & poly(D-lactide) & SIS & poly(styrene- $b$-isoprene- $b$-styrene) \\
\hline PDLLA & poly(D,L-lactide) & TEM & Transmission Electron Microscopy \\
\hline $\begin{array}{l}\text { PDMA- } \\
\text { EMA }\end{array}$ & $\begin{array}{l}\text { poly-[2-(dimethylamino)ethyl } \\
\text { methacrylate] }\end{array}$ & THF & tetrahydrofuran \\
\hline PDMS & polydimethylsiloxane & TPEs & thermoplastic elastomers \\
\hline PEO & poly(ethylene oxide) & UF & ultrafiltration \\
\hline PEOT & poly(ethylene oxide)terephthalate & WAXS & Wide Angle X-ray Scattering \\
\hline
\end{tabular}

\section{Introduction}

With the emergence of nanotechnology, fibres with diameters down to tens of nanometres make themselves attractive for a wide variety of applications ranging from tissue engineering scaffolds ${ }^{2}$ to energy harvesting devices ${ }^{3}$ and protective clothing. ${ }^{4}$ Compared to other nanofibre fabrication techniques, electrospinning ${ }^{5-9}$ is the most facile and highly versatile approach that allows continuous nanofibres with diameters ranging from $2 \mathrm{~nm}$ to several micrometres to be produced on a large scale. This method has attracted tremendous interest in both academia and industry since the early 1990s due to its ability to produce novel nanofibres and nonwoven mats with high surface area-to-volume ratio and controllable porous structures. In electrospinning, ${ }^{8,10,11}$ a high electric field is applied to the liquid droplet. A charged 
jet is ejected from the tip of the nozzle (or needle) when the electrostatic repulsion overcomes surface tension to sufficiently stretch the droplet. The solvent begins to evaporate as the jet then travels towards a grounded electrode, where the resultant fibres are collected. During the process, many factors, including solution properties (e.g., viscosity, concentration, electrical conductivity, etc.), processing conditions (e.g., voltage, flow rate, collecting distance, etc.), and environmental conditions (temperature, humidity, atmospheric pressure) have varying influence on the diameter, organization/arrangement and surface morphology of the final product. ${ }^{7,9,12}$ Through adjustment of these electrospinning parameters, the desired characteristics of the nanofibres, such as high surface area-to-volume ratio, interconnected porosity with tuneable pore size or structures similar to the extracellular matrix $(\mathrm{ECM})$ can be obtained. So far, this technique has been widely used to construct nanofibres for sensors, ${ }^{13}$ filtration, ${ }^{14}$ drug delivery, ${ }^{15}$ and tissue engineering. ${ }^{16}$ A variety of natural and synthetic polymers have been electrospun into nanofibres and nonwoven mats, such as collagen, ${ }^{17}$ gelatin, ${ }^{18}$ silk fibroin, ${ }^{19}$ PLA, ${ }^{20-22}$ cellulose derivatives ${ }^{23}$, polyurethanes, ${ }^{24}$ polystyrenics, ${ }^{25,26}$ and so on.

In parallel, as an indispensable class of polymeric materials, BCPs, containing two or more chemically distinct and covalently linked homopolymers, represent an intriguing class of macromolecules. ${ }^{27,28}$ They have been widely utilized as thermoplastic elastomers, ${ }^{29}, 30$ adhesives ${ }^{31}$ and stimuli-responsive materials. ${ }^{32-36}$ Moreover, due to the inherent thermodynamic immiscibility of different polymer segments, BCPs are known to self-assemble ${ }^{37-40}$ into various periodic nanoscale structures (such as spheres, cylinders and lamellae), depending on the relative volume fractions of the blocks, the total degree of polymerization and the Flory-Huggins parameter (a temperature-dependent interaction parameter that describes the miscibility between the disparate monomer segments). This behaviour is driven by an unfavourable mixing enthalpy coupled with a sufficiently small de-mixing entropic penalty, which has been extensively studied to create highly ordered, three-dimensional, structural hierarchies with characteristic feature sizes as self-organizing materials via a 
"bottom-up" approach. Thus, the vast applications of BCPs arise from not only the traditional advantages of polymeric materials and control of functionality, but also microphase separation control over the nanometre length scale and morphology.

The unique behaviour and properties of BCPs have been studied in the melt/bulk, ${ }^{41}$ as thin films ${ }^{42-46}$ and in solution ${ }^{47}$. A plethora of BCPs have been used to fabricate nanofibres with controllable, exquisite properties. Here, a comprehensive review is provided with a focus on the various strategies used to electrospin BCPs and exploit their behaviour in nonwoven fabrics. The review covers the applications of functional BCP nanofibres as biomaterials, separation membranes, sensors and electronic materials (Figure 1). Subsequently, a small selection of further applications is covered before we summarize and provide an outlook on this exciting field.

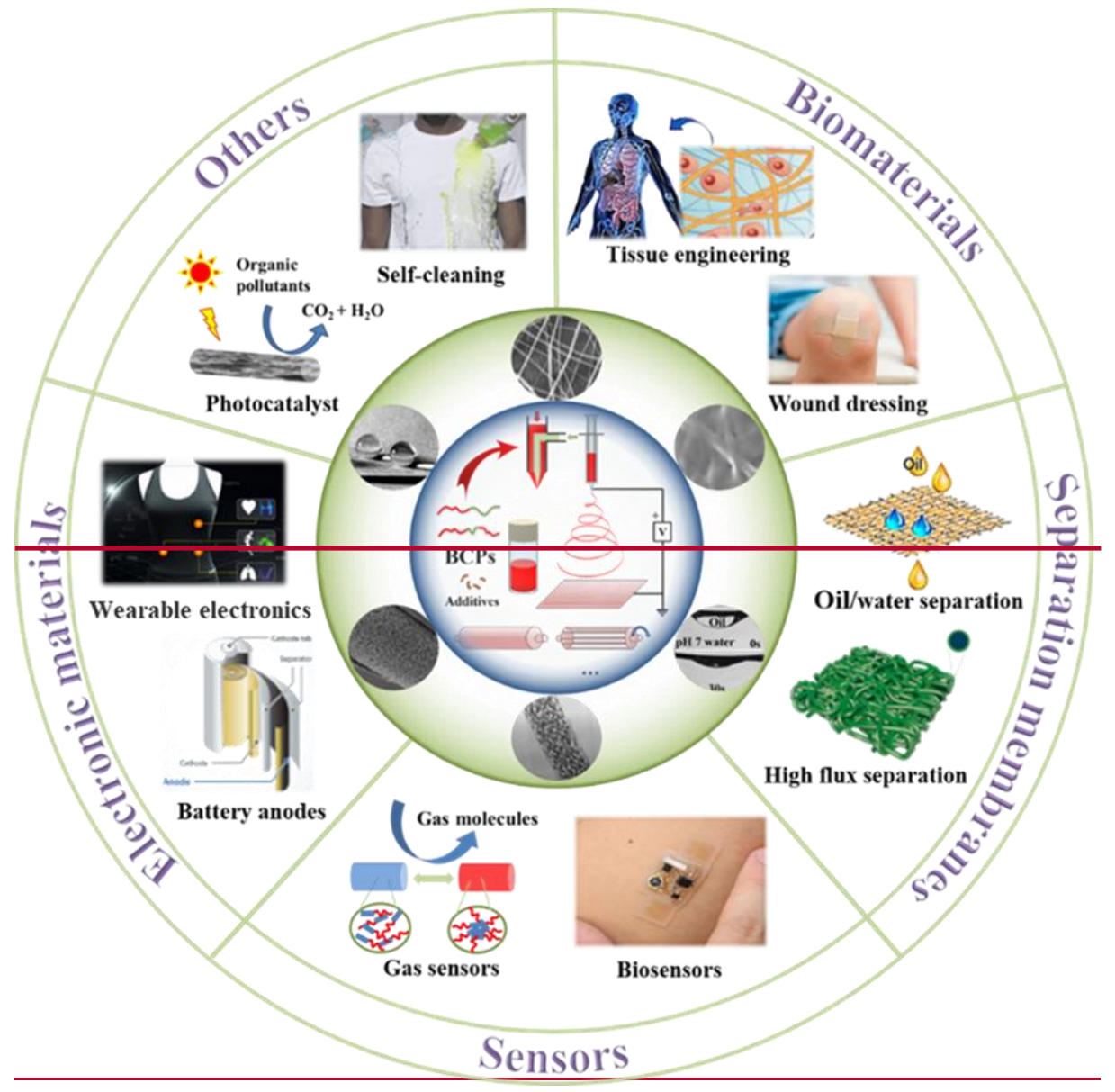




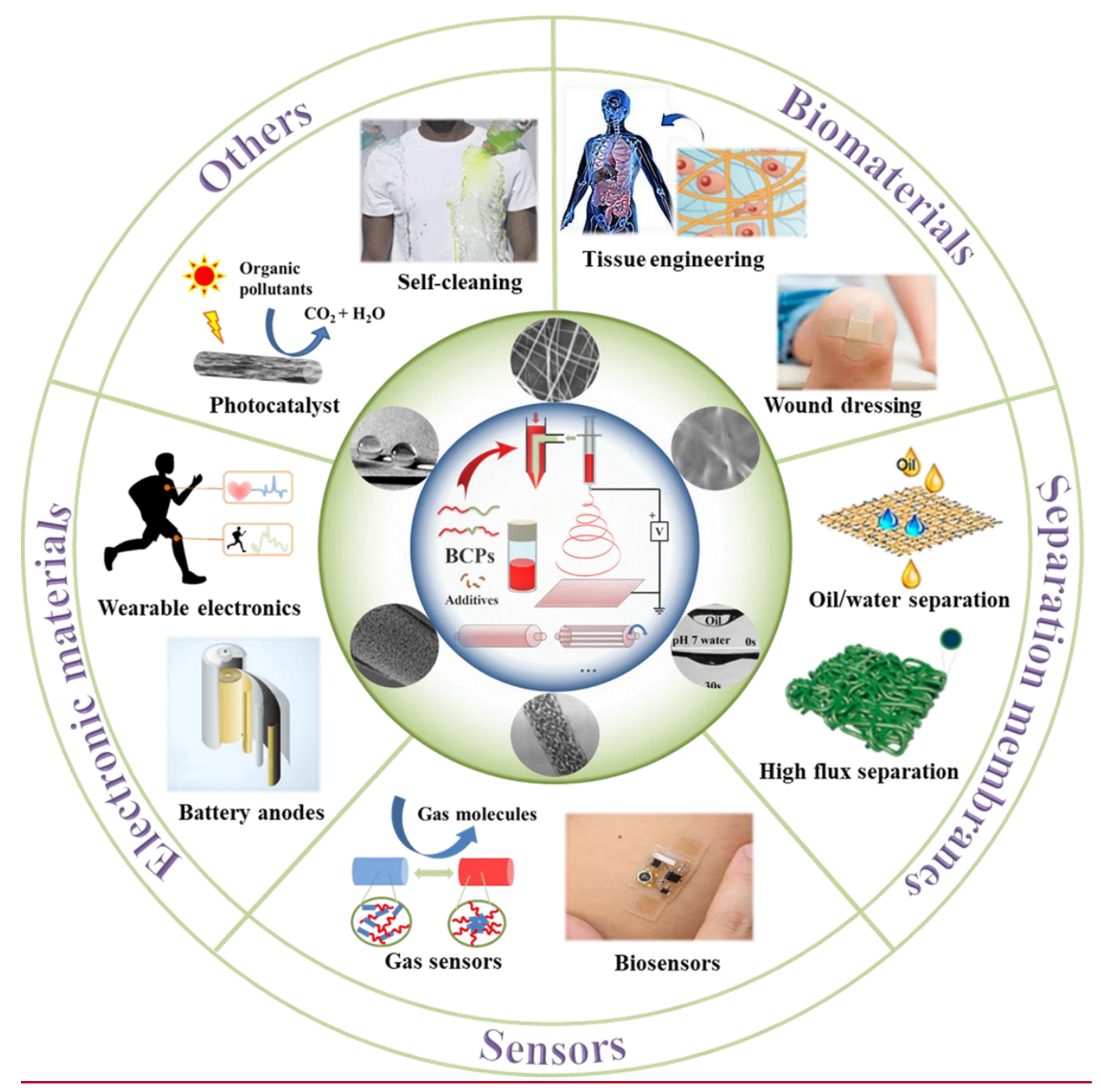

Figure 1. The various properties and applications of BCP nanofibres through the manipulation of electrospinning. Partial images adapted from Ref.[29],Copyright 2005; Ref.[40a],Copyright 2016. Partial images adapted with permission from Ref.14. (Copyright 2018 American Chemical Society) and the related work mentioned below.

\subsection{Properties}

The systematic development of BCPs is intimately linked with functional blocks and well-defined equilibrium structures from self-assembled domains. BCP nanofibres not only retain the advantages of BCPs but also combine the structural features (high surface area) of nanofibres to boost their performance in a variety of modes.

Typically, BCPs consisting of rubbery and glassy segments can create TPEs which show the advantages of both rubbery and plastic (non-elastic) materials, such as 
greater toughness and strength, and the specific conditions in processing. For example, SBS, ${ }^{48-50}$ SI, SIS ${ }^{51,52}$ and SEBS ${ }^{49,53}$ have been electrospun into submicron fibres with good fibre morphology and mechanical properties. Crucially, after annealing, the fibres exhibited a more significant contrast between the rubbery and glassy segments, indicating stronger microphase segregation (shown in Figure 2). ${ }^{48,52-54}$
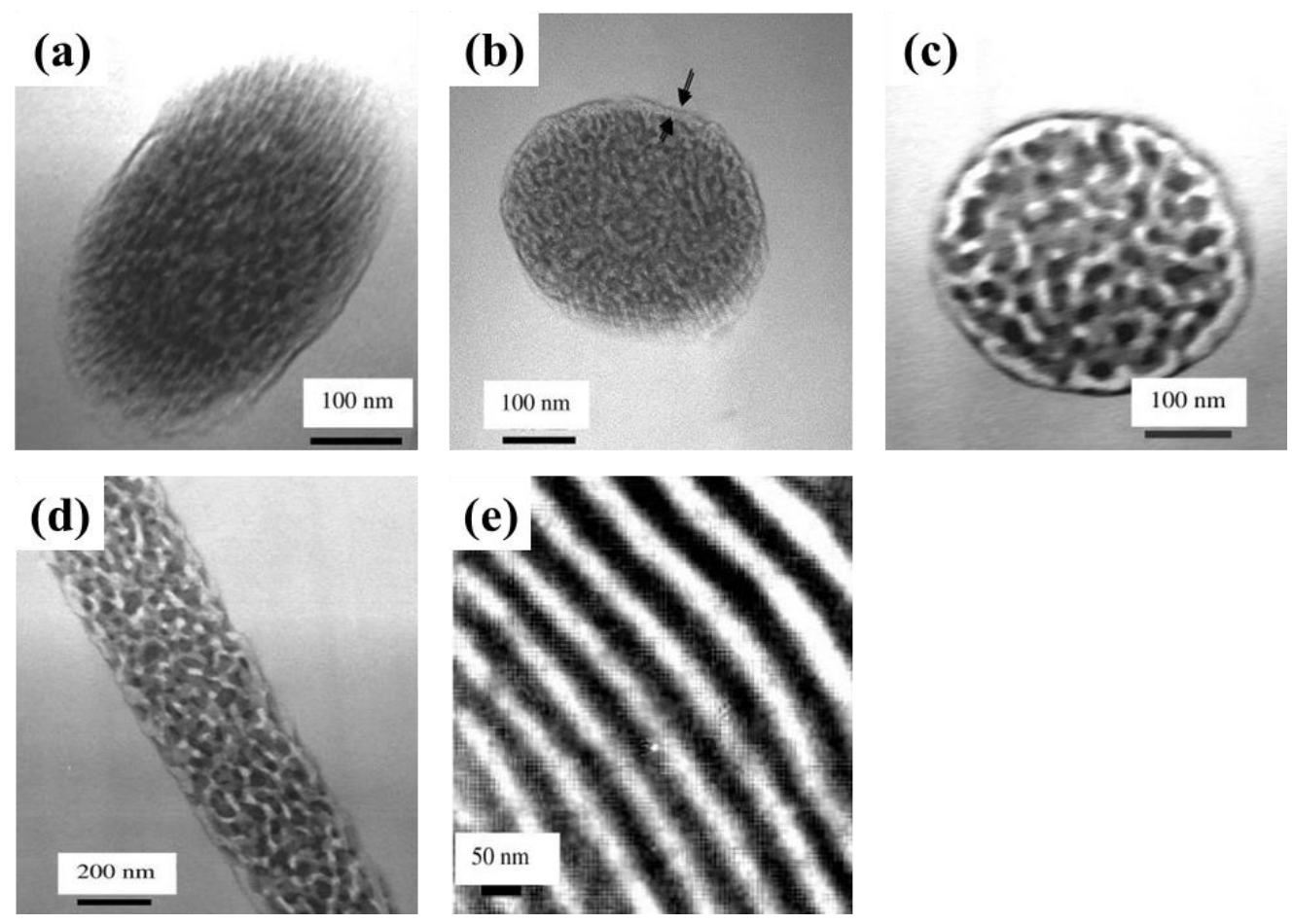

Figure 2. TEM images of self-assembled structures in electrospun PS- $b$-PI fibres: (a, b) Two cross sections with different domain structures of fibres spun from $25 \mathrm{wt} \%$ solution in THF; (c) A cross section of a fibre annealed at $90{ }^{\circ} \mathrm{C}$ for $12 \mathrm{~h}$; (d) The fibre axis annealed at $90{ }^{\circ} \mathrm{C}$ for $12 \mathrm{~h}$ and (e) a film cast from $10 \mathrm{wt} \%$ solution in THF. ${ }^{54}$ Images are adapted with permission from Ref. 54. (Copyright 2006 American Chemical Society)

Different assembled structures within nanofibres also had been investigated. ${ }^{55} \mathrm{~A}$ series of PMMA- $b$-PS BCPs with various molecular weights and composition was synthesized, and electrospun into nanofibres. After selective solvent annealing, these $\mathrm{BCP}$ nanofibers with long-range ordered nanostructures exhibited a preferred domain orientation that is perpendicular to the fibre axis. With increasing PS volume fraction, highly ordered PS cylinders, lamellae, or PMMA cylinders were formed, respectively, 
within the nanofibers (Figure 3). After removal of a sacrificial block, the preferred domain orientation in BCP nanofibers can enable the creation of porous fibrous matrices that have potential application in fields such as catalysis, separation or filtration.

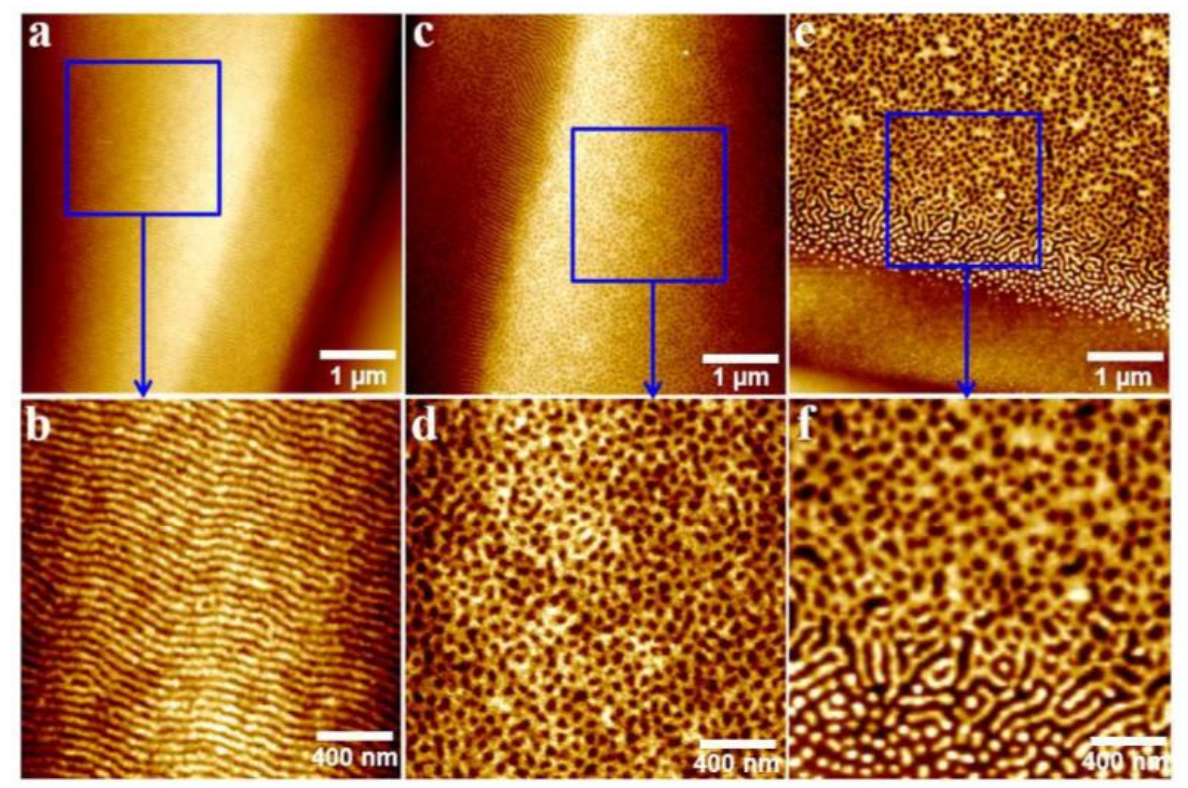

Figure 3. AFM height images of PMMA- $b$-PS fibres after solvent annealing in chloroform vapor for $31 \mathrm{~h}$. The PS volume fraction of (a and b) 41\%; (c and d) 51\%; and (e and f) $66 \%$, respectively. ${ }^{55}$ Images are adapted with permission from Ref. 55. (Copyright 2018 Royal Society of Chemistry)

The microphase separation of block copolymers has a significant influence on the wetting behaviour of nanofibrous surfaces. Besides the rough structure of the fibrous membrane surface and hydrophobic polymer segments within the fibres, the formation of ordered nanodomains with hydrophobicity enable the superhydrophobicity of fibrous membranes readily controlled after suitable post treatment. Ma et $a l^{56}$ reported that superhydrophobic microphase separated nanofibres could be obtained by electrospinning PS- $b$-PDMS/PS blends. Microphase separation within the fibres was confirmed by DSC and the lower surface tension in combination with the roughness provided by electrospinning account for the superhydrophobicity of the fibre mats. Furthermore, a series of diblock and triblock copolymers based on PS with fluorinated blocks (e.g., FMA, FS, EMS and FSF) were 
synthesized to enhance the hydrophobicity of the nanofibrous surface. ${ }^{57,}{ }^{58} \mathrm{By}$ coupling the surface enrichment of low surface energy blocks with the surface roughness of nonwoven mats, in addition to the distinct phase segregated nature of BCP fibres, superhydrophobic fabrics can be successfully fabricated. SEM images of the fibres and photographic images of water droplets on the fabrics are shown in Figure 4, illustrating the superhydrophobicity of the PS- $b$-PDMS/PS fibrous mats.
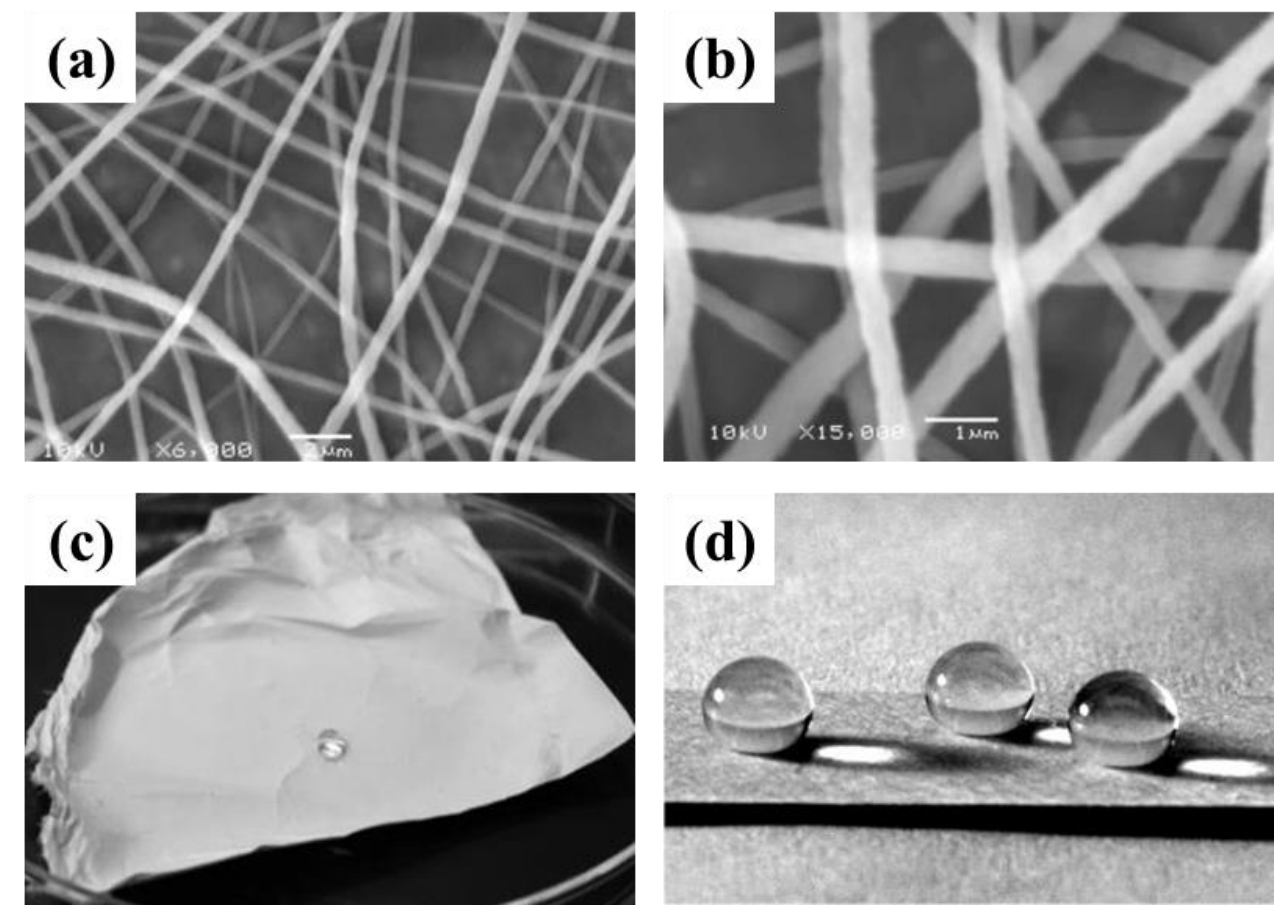

Figure 4. Superhydrophobic PS- $b$-PDMS/PS fibrous mats. SEM images of the electrospun fibres at (a) 6000x magnification (scale bar $=2 \mu \mathrm{m}$ ) and (b) $15000 \times$ magnification (scale bar $=1 \mu \mathrm{m}$ ); (c) Free-standing mat composed of the PS- $b$-PDMS/PS electrospun fibres with a water droplet standing proud upon it; (d)

Several water droplets on the mat to demonstrate the high water contact angle. ${ }^{56,57}$ Images are adapted with permission from Ref. 56. (Copyright 2005 American Chemical Society)

In contrast to superhydrophobic materials, amphiphilic block copolymers (ABCs) consisting of a hydrophilic block that is chemically tethered to a hydrophobic block have been used extensively to control the hydrophobicity/hydrophilicity of membrane surfaces. Notably, PPO, having some hydrophilic character, has been combined with 
polystyrene to prepare PS- $b$-PPO to tailor the wettability of the surface ${ }^{59}$ Poloxamer, also known as Pluronic $\AA$, composed of a central (more) hydrophobic chain of PPO flanked by two hydrophilic chains of PEO, are the most widely studied as nonionic surfactants. Pluronic F108, ${ }^{60}$ Pluronic F127 ${ }^{61,62}$ (where F indicates that the material is a flaky solid, the first two digits multiplied by 100 give the approximate molar mass of the PPO block, and the last digit multiplied by 10 gives the approximate PEO content as a percentage) were both solution-blended with hydrophobic (PLGA and SEBS prior to electrospinning, in a bid to fabricate nanofibrous membranes with enhanced hydrophilicity. In biomedical fields, similar efforts have been made to control the biodegradation rate of nanofibres or in vivo behaviour of the membranes. A PEO segment is often introduced into a hydrophobic polymer system, such as PDLA, PLLA, PCL and so on, to improve the hydrophilicity, pliability, retention time (in the body) and degradability of the biodegradable material. Diblock copolymer PEO- $b$-PCL ${ }^{63}$ and PEO- $b$-PLA ${ }^{64}$ multiblock copolymers based on PLLA- $b$-PEO ${ }^{65}$ were synthesized and the physico-chemical properties and in vitro degradation behaviour of the nonwoven nanofibre membranes were investigated. BCPs comprising a hydrophilic POEOMA, PMPC, or PDMAEMA block and a hydrophobic PLLA block were used to increase surface hydrophilicity and cell adhesion (when compared to PDLLA fibres) by Viswanathan and co-workers. They also exploited the microphase separation of the incompatible hydrophilic PMPC and POEGMA blocks at the solid-air interface of PDLLA fibres and the effect on controlling cell behaviour (Figure 5). ${ }^{66}$ All of these examples neatly demonstrate how the incorporation and manipulation of BCPs can allow us to finely control the surface properties of a material through molecular design. 
(a)

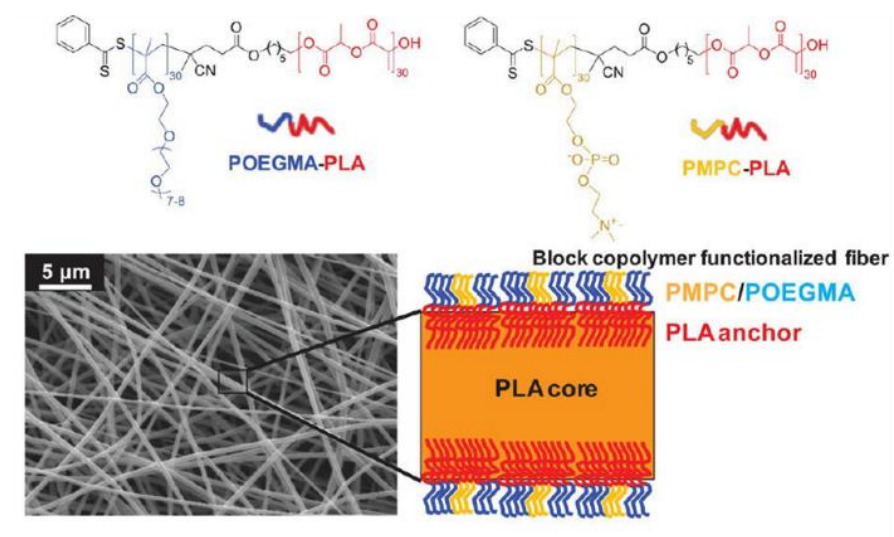

(b)

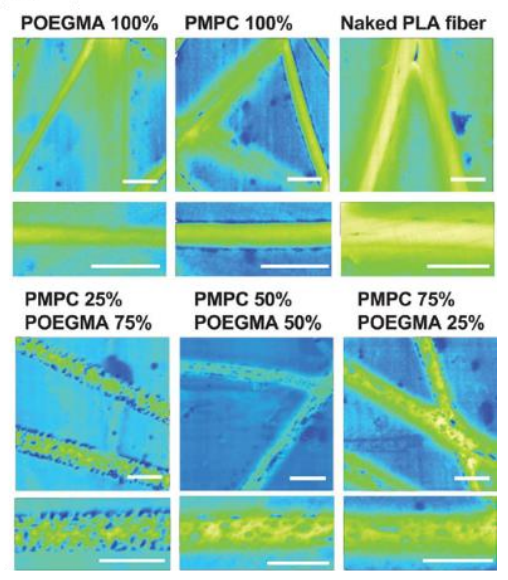

Figure 5. Functionalized amphiphilic block copolymer fibres used for cell adhesion. (a) Surface functionalization of PDLLA electrospun fibres by incorporation of POEGMA- $b$-PLA and PMPC- $b$-PLA amphiphilic linear diblock copolymers. (b) POEGMA- $b$-PLA and PMPC- $b$-PLA diblock copolymers mixed in various molar ratios inducing microphase separation at the solid-water interface. Fibres were imaged by SEM using BEI. Scale bar $=500 \mathrm{~nm}$ in each case. ${ }^{66}$-Images are adapted with permission from Ref. 66. (Copyright 2015 American Chemical Society)

Many responsive monomers have been synthesized into BCPs to combine other polymers for expanding the properties of material. Nanofibers comprising responsive BCPs which can not only keep the intrinsic characteristics of nanofibers, but can also switch their chemical and physical properties in response to stimuli, such as temperature, $\mathrm{pH}$, light and so on, have been electrospun for many smart devices. For example, PNIPAM is an extensively studied thermoresponsive polymer, particularly used for biomedical applications. Antti et al. ${ }^{67}$ studied the wetting behaviour and morphology of thermally responsive amphiphilic PS- $b$-PNIPAM- $b$-PS fibrous membranes. Nanofibrous membranes of BCPs consisting of PNIPAM ${ }^{68,69}$ exhibit temperature switchable wettability that can be maintained and controlled under appropriate conditions. Furthermore, BCPs can also be incorporated with other stimuli-responsive blocks, such as a weak polybasic segment (e.g. P4VP), ${ }^{70,71}$ and can therefore endow nanofibrous membranes with two switchable wettability states (using temperature or $\mathrm{pH}$ ) because of the protonation and deprotonation of the 
pyridyl groups (Figure 6). In other work, ${ }^{72}$ a symmetrical ABA triblock copolymer, PMMA- $b$-PDEA- $b$-PMMA was electrospun to create $\mathrm{pH}$-responsive fibre membranes, where the central block was pH-responsive. Non-responsive, hydrophobic flanking groups were used to create a physically crosslinked network, bound by the self-assembling nature of the block copolymer. The use of noncovalent, self-assembled crosslinks allows such materials to be reprocessed (i.e. electrospun again from solution), unlike networks formed from chemical crosslinks, where irreversible mechanical failure will render the material consumed.

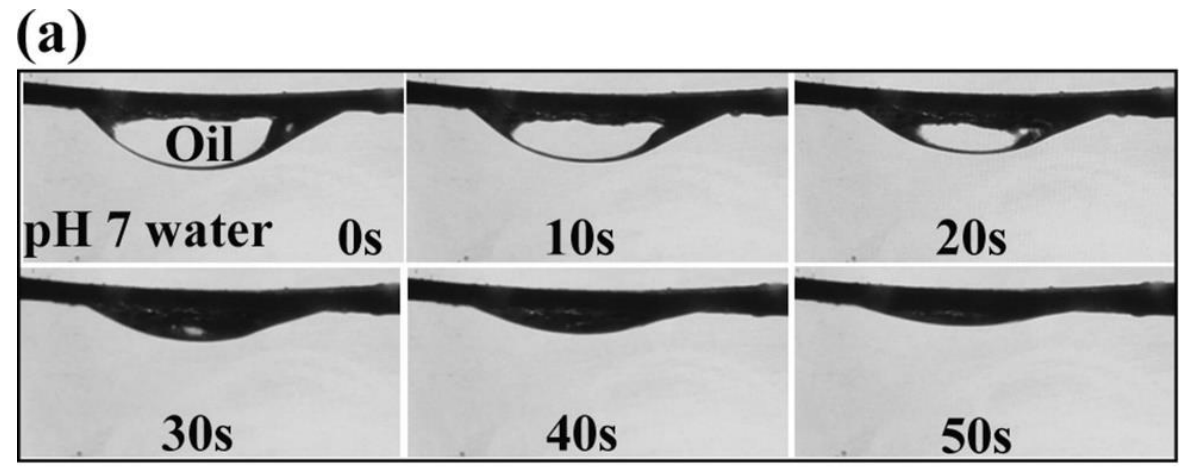

(b)

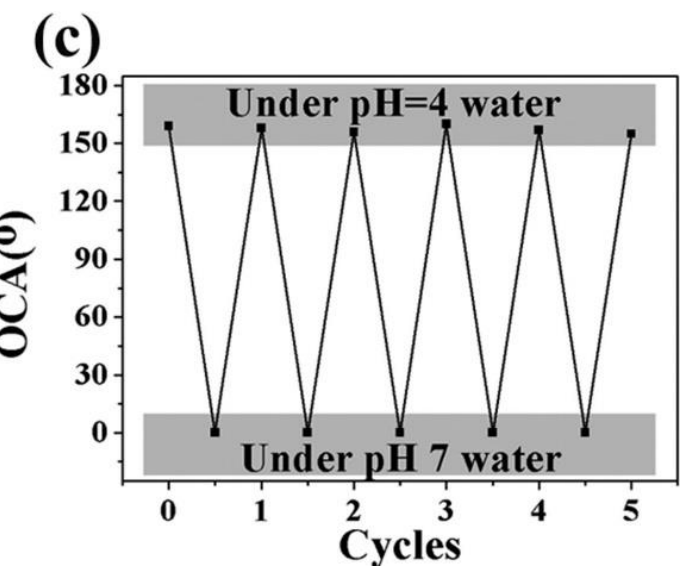

Figure 6. PDMS-b-P4VP mat exhibited good pH-induced oil/water wettability. Oil wettability of the as-prepared polymer mat under an aqueous medium with different $\mathrm{pH}$ values. (a) Dynamic images of an oil droplet (hexane) spreading over the mat within $50 \mathrm{~s}$ under $\mathrm{pH} 7$ water. (b) An oil droplet on the mat under $\mathrm{pH} 4$ water with an OCA of approximately $158^{\circ}$. (c) Reversible oil wettability of the as-prepared mat in different aqueous media. ${ }^{70}$ Images are adapted with permission from Ref. 70. (Copyright 2016 American Chemical Society) 
Electrospinning had also been utilized as a template (top-down) to fabricate ultrathin fibres and self-assembly (bottom-up) of BCPs to construct ordered microstructures. Compared to normal polymer nanofibers whose morphology only can be controlled by adjusting processing parameter of electrospinning and ambient conditions, BCP nanofibers with controlled domain orientations offer more control for potential mesoporous structures. $\mathrm{Li}$ and co-workers ${ }^{73}$ synthesized novel cigar-like $\mathrm{TiO}_{2}$ fibres with an outer-shell and inner-continuous-pore structure by coupling the self-assembly of PS- $b$-PEO containing titanium tetraisopropoxide (a precursor of $\mathrm{TiO}_{2}$ ) with the electrospinning technique and investigated the structure control in these nanofibres in detail (Figure 7). ${ }^{74}$ Fibre composites containing these amphiphilic BCPs were electrospun prior to carbonization to obtain hierarchical structures. ${ }^{75,76}$ The Pluronic ${ }^{\circledR}$ triblock copolymer P123 (where P denotes a paste-like physical appearance) which was used as a structure-directing agent, and poly(vinyl pyrrolidone) (PVP) which was used as a fibre template, were subjected to electrospinning to fabricate continuous ordered mesoporous silica nanofibres. ${ }^{77-79}$
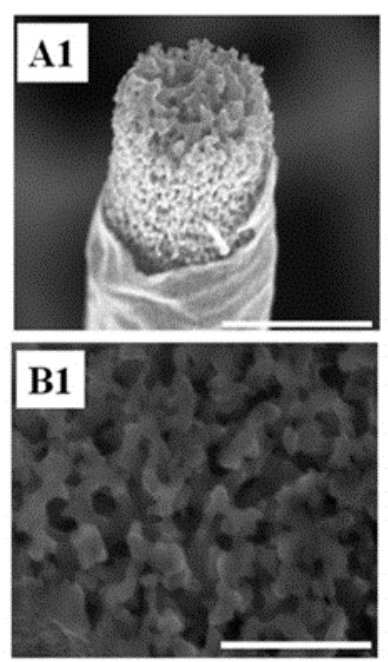
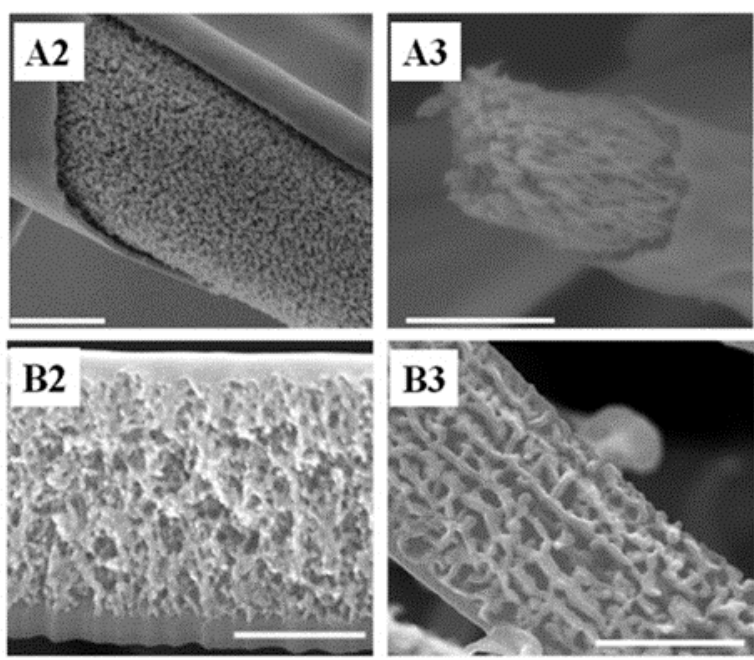

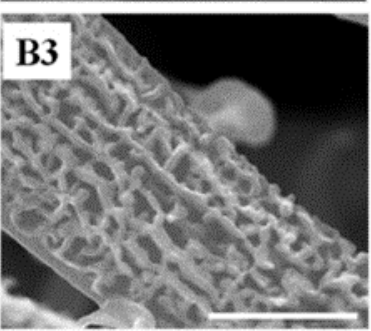

Figure 7. Novel cigar-like $\mathrm{TiO}_{2}$ fibres synthesized through the use of different volume fractions of PS- $b$-PEO. SEM images of $\mathrm{TiO}_{2}$ fibres using (A) PS- $b$-PEO $\left(M_{\mathrm{n}} \sim\right.$ $38,000-b-102,000)$ and (B) $\mathrm{PS}_{59000}-b-\mathrm{PEO}_{31000}\left(M_{\mathrm{n}} \sim 59,000-b-31,000\right)$. Scale bars are $500 \mathrm{~nm}$ (A1, B1, A3), $300 \mathrm{~nm}$ (A2, B2), and $400 \mathrm{~nm}$ (B3). ${ }^{74} \underline{\text { Images are adapted with }}$ permission from Ref. 74. (Copyright 2016 American Chemical Society) 
BCPs have been used to deliver spatial control over NPs within nanofibres by exploiting the self-assembly of BCPs with partitioning of NPs within block-specific domains. The effectiveness of these NPs within the $\mathrm{BCP}$ composite nanofibres were enhanced significantly. Kalra et al. ${ }^{80}$ prepared coaxial nanofibres with a core of PS- $b$-PI/magnetite NPs and an outer a silica shell to produce nanocomposite fibres with superparamagnetic behaviour. In further work, the same group used a similar approach to guide the placement of other functional NPs by triaxial/coaxial electrospinning. ${ }^{81}$

In the past few decades, various BCP fibres with precisely controlled morphology and properties have been successfully gained through tuning of the electrospinning parameters and functional segments of BCPs, which has broadened the application of electrospun fibres in many fields. The microphase separation-induced ordered domains in BCP nanofibers can equip the porous fibrous membranes with shortened diffusion length for ions, gases, and other species after removing a sacrificial block, provide control over the surface energy (such as superhydrophobicity), and construct mesoporous nanofibrous composites as templates for nanoparticles, as discussed in the next chapter.

\subsection{Applications}

Most research emphasis of the reported BCP nanofibres made by electrospinning has been placed on the physical properties, morphology and topology of nonwoven mats and the behaviour of microphase separation within the nanofibres. The various outstanding BCP nanofibre properties (determined by the molecular composition, electrospinning parameters and post-deposition treatment), such as mechanical properties, superhydrophobicity, amphiphilicity, stimuli response, and mesoporosity, have been studied and further exploited for a series of applications. Here, electrospun $\mathrm{BCP}$ nanofibres used for tissue engineering, drug delivery, wound dressings, oil/water separation membranes, ion-exchange membranes, protein adsorption, gas sensors, biosensors, electronic materials and other applications are discussed in detail. 
Table 1 summarizes the $\mathrm{BCP}$ nanofibres reported in recent decades, describing the BCP used, research context and related application(s). In order to assist experimentalists, Table 1 also lists the solvent(s) used, type of electrospinning employed, diameter of nanofibers and the post treatment of nanofibres.

Table 1 Summary of reported work on BCP nanofibres across the literature.

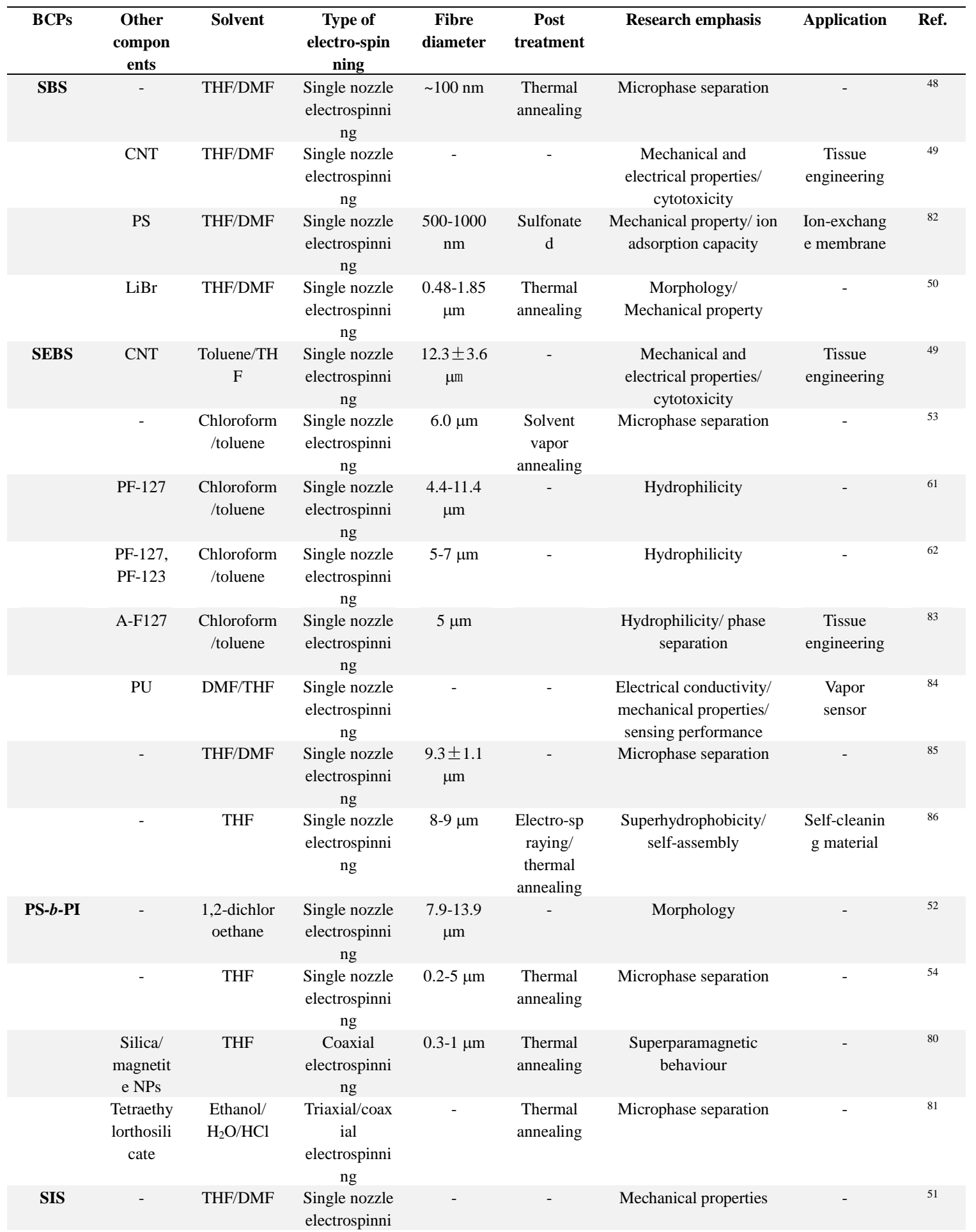




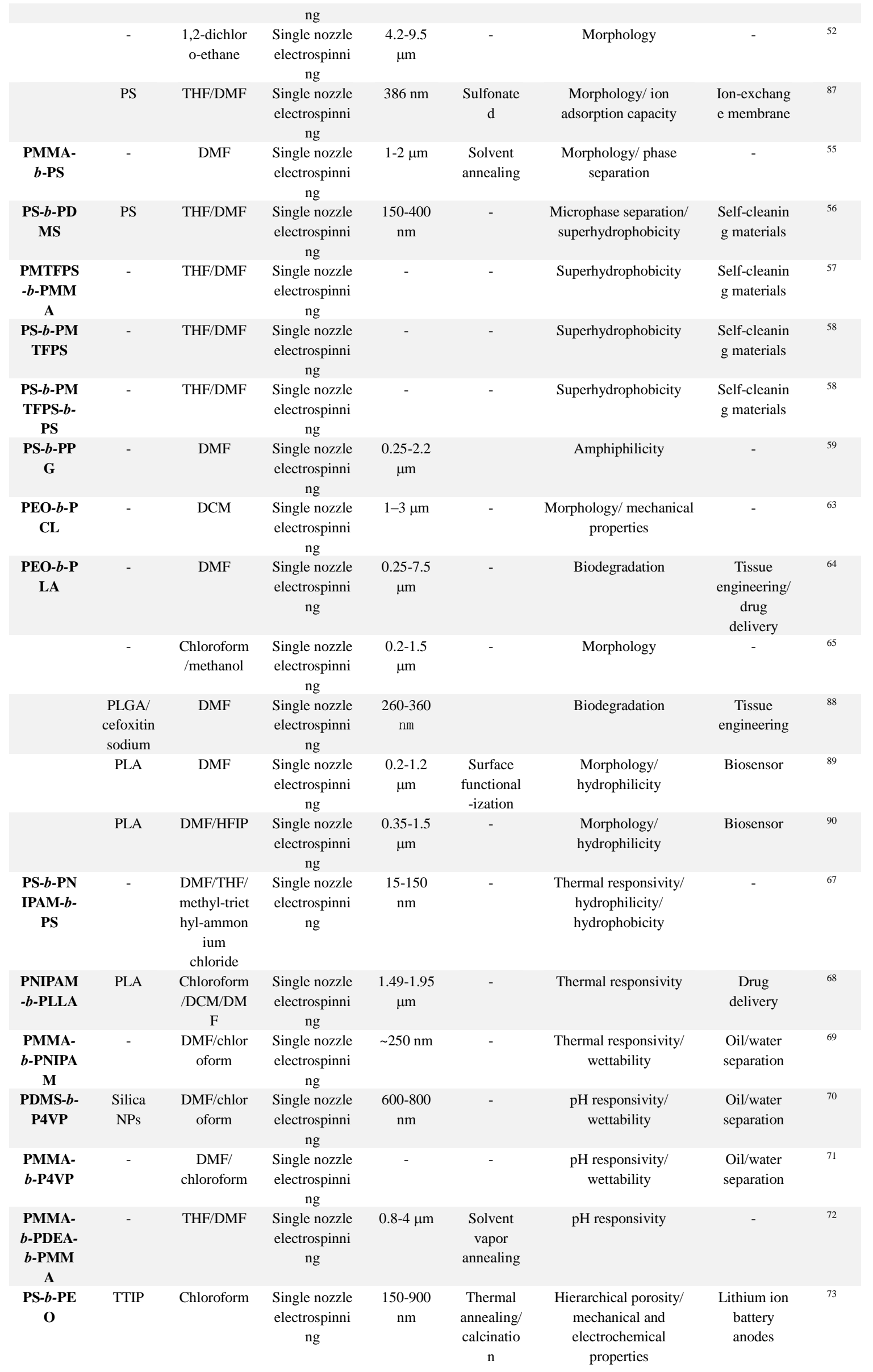




\begin{tabular}{|c|c|c|c|c|c|c|c|c|}
\hline & TTIP & $\begin{array}{l}\text { Chloroform } \\
\text { /DMF }\end{array}$ & $\begin{array}{c}\text { Single nozzle } \\
\text { electrospinni } \\
\text { ng }\end{array}$ & $\begin{array}{l}150-4000 \\
\mathrm{~nm}\end{array}$ & $\begin{array}{c}\text { Thermal } \\
\text { annealing/ } \\
\text { calcinatio } \\
\mathrm{n}\end{array}$ & $\begin{array}{c}\text { Structure/ photocatalysis } \\
\text { performance }\end{array}$ & Catalysis & 74 \\
\hline $\begin{array}{c}\text { PMPEG } \\
\text { MA- } b-P \\
\text { BA }\end{array}$ & $\begin{array}{l}\mathrm{Ni} / \mathrm{NiO} / \\
\mathrm{MnO}_{\mathrm{x}}\end{array}$ & THF & $\begin{array}{c}\text { Single nozzle } \\
\text { electrospinni } \\
\text { ng }\end{array}$ & $\begin{array}{l}600-1200 \\
\mathrm{~nm}\end{array}$ & $\begin{array}{c}\text { Calcinatio } \\
\mathrm{n}\end{array}$ & $\begin{array}{c}\text { Hierarchical } \\
\text { porosity/cooperative } \\
\text { assembly/ electrical } \\
\text { conductivity }\end{array}$ & $\begin{array}{c}\text { Lithium ion } \\
\text { battery } \\
\text { anodes }\end{array}$ & 75 \\
\hline \multirow[t]{2}{*}{$\begin{array}{c}\text { PEOT/P } \\
\text { BT }\end{array}$} & $\begin{array}{l}\text { Dexamet } \\
\text { ha-sone }\end{array}$ & $\begin{array}{l}\text { Chloroform } \\
\text { /ethanol }\end{array}$ & $\begin{array}{c}\text { Single nozzle } \\
\text { electrospinni } \\
\text { ng }\end{array}$ & $\begin{array}{c}0.51-2.15 \\
\mu \mathrm{m}\end{array}$ & - & $\begin{array}{l}\text { Mechanical properties/ } \\
\text { drug encapsulation }\end{array}$ & $\begin{array}{c}\text { Tissue } \\
\text { engineering/ } \\
\text { drug } \\
\text { delivery }\end{array}$ & 91 \\
\hline & - & Chloroform & $\begin{array}{c}\text { Single nozzle } \\
\text { electrospinni } \\
\text { ng }\end{array}$ & $1-4 \mu \mathrm{m}$ & - & Biocompatibility & $\begin{array}{c}\text { Tissue } \\
\text { engineering }\end{array}$ & 92,93 \\
\hline \multirow[t]{3}{*}{ PCEC } & $\begin{array}{l}\text { Nano } \\
\mathrm{Fe}_{3} \mathrm{O}_{4}\end{array}$ & DCM & $\begin{array}{c}\text { Single nozzle } \\
\text { electrospinni } \\
\text { ng }\end{array}$ & $1.23 \mu \mathrm{m}$ & - & $\begin{array}{l}\text { Biodegradation/ } \\
\text { hydrophilicity }\end{array}$ & $\begin{array}{c}\text { Tissue } \\
\text { engineering }\end{array}$ & 94 \\
\hline & $\begin{array}{l}\text { Curcumi } \\
\mathrm{n}\end{array}$ & DCM & $\begin{array}{l}\text { Single nozzle } \\
\text { electrospinni } \\
\text { ng }\end{array}$ & $\sim 4 \mu \mathrm{m}$ & - & $\begin{array}{l}\text { Biodegradation/ } \\
\text { hydrophilicity }\end{array}$ & $\begin{array}{c}\text { Tissue } \\
\text { engineering }\end{array}$ & 95,96 \\
\hline & $\begin{array}{l}\text { Hydroxy } \\
\text {-apatite }\end{array}$ & DCM & $\begin{array}{c}\text { Single nozzle } \\
\text { electrospinni } \\
\text { ng }\end{array}$ & $0.6-1 \mu \mathrm{m}$ & - & $\begin{array}{l}\text { Biocompatibility/ } \\
\text { hydrophilicity }\end{array}$ & $\begin{array}{c}\text { Tissue } \\
\text { engineering }\end{array}$ & $97-99$ \\
\hline $\begin{array}{c}\text { PCL- } \\
b \text {-PTHF- } \\
b \text {-PCL }\end{array}$ & PCL & $\begin{array}{l}\text { Chloroform } \\
\text { /methanol }\end{array}$ & $\begin{array}{c}\text { Single nozzle } \\
\text { electrospinni } \\
\text { ng }\end{array}$ & $0.4-5 \mu \mathrm{m}$ & - & $\begin{array}{c}\text { Mechanical properties/ } \\
\text { hydrophilicity }\end{array}$ & $\begin{array}{c}\text { Tissue } \\
\text { engineering }\end{array}$ & 100 \\
\hline $\begin{array}{l}\text { PMMA- } \\
b \text {-MA }\end{array}$ & PCL & THF/DMF & $\begin{array}{l}\text { Single nozzle } \\
\text { electrospinni } \\
\text { ng }\end{array}$ & $350 \mathrm{~nm}$ & & $\begin{array}{c}\text { Surface properties/ } \\
\text { mechanical properties }\end{array}$ & $\begin{array}{l}\text { Wound } \\
\text { dressing }\end{array}$ & 101 \\
\hline $\begin{array}{l}\text { PVP- } b-P \\
\text { CL }\end{array}$ & PCL & THF/DMF & $\begin{array}{c}\text { Single nozzle } \\
\text { electrospinni } \\
\mathrm{ng}\end{array}$ & $\begin{array}{c}407.8- \\
438.3 \mathrm{~nm}\end{array}$ & - & $\begin{array}{l}\text { Hydrophilicity/ } \\
\text { biocompatibility }\end{array}$ & $\begin{array}{c}\text { Tissue } \\
\text { engineering }\end{array}$ & 102 \\
\hline \multirow[t]{12}{*}{$\begin{array}{l}\text { Poloxam } \\
\text { er }\end{array}$} & $\begin{array}{l}\mathrm{SiO}_{2} / \mathrm{PV} \\
\mathrm{P}\end{array}$ & Ethanol & $\begin{array}{c}\text { Single nozzle } \\
\text { electrospinni } \\
\text { ng }\end{array}$ & $\begin{array}{l}100-1000 \\
\mathrm{~nm}\end{array}$ & $\begin{array}{c}\text { Calcinatio } \\
\mathrm{n}\end{array}$ & $\begin{array}{l}\text { Nanoporous structure/ } \\
\text { adsorption capacity }\end{array}$ & $\begin{array}{c}\text { Dye } \\
\text { absorption }\end{array}$ & 76 \\
\hline & $\begin{array}{c}\mathrm{SiO}_{2} / \mathrm{PV} \\
\mathrm{P}\end{array}$ & Ethanol & $\begin{array}{c}\text { Single nozzle } \\
\text { electrospinni } \\
\text { ng }\end{array}$ & $\begin{array}{c}200-300 \\
\mathrm{~nm}\end{array}$ & $\begin{array}{c}\text { Calcinatio } \\
\mathrm{n}\end{array}$ & Nanoporous structure & - & 79 \\
\hline & - & Ethanol & $\begin{array}{c}\text { Single nozzle } \\
\text { electrospinni } \\
\text { ng }\end{array}$ & $470 \mathrm{~nm}$ & - & $\begin{array}{l}\text { Hierarchically porosity/ } \\
\text { cell proliferation }\end{array}$ & $\begin{array}{c}\text { Tissue } \\
\text { engineering }\end{array}$ & $\begin{array}{l}77 \\
78\end{array}$ \\
\hline & $\begin{array}{l}\text { PLCL/ } \\
\text { dextran/ } \\
\text { gelatin }\end{array}$ & THF/DMF & $\begin{array}{c}\text { Single nozzle } \\
\text { electrospinni } \\
\text { ng }\end{array}$ & $\begin{array}{l}855.77-14 \\
26.92 \mathrm{~nm}\end{array}$ & - & $\begin{array}{l}\text { Hydrophilicity/ } \\
\text { mechanical properties }\end{array}$ & $\begin{array}{c}\text { Tissue } \\
\text { engineering }\end{array}$ & 103 \\
\hline & $\begin{array}{l}\text { Silk } \\
\text { fibroin }\end{array}$ & HFIP & $\begin{array}{c}\text { Single nozzle } \\
\text { electrospinni } \\
\text { ng }\end{array}$ & $\begin{array}{c}2.4-5.9 \\
\mu \mathrm{m}\end{array}$ & - & Hydrophilicity & & 104 \\
\hline & ADSCs & HFIP & $\begin{array}{c}\text { Single nozzle } \\
\text { electrospinni } \\
\text { ng }\end{array}$ & - & - & & $\begin{array}{l}\text { Wound } \\
\text { dressing }\end{array}$ & 105 \\
\hline & PLLA & Chloroform & $\begin{array}{c}\text { Single nozzle } \\
\text { electrospinni } \\
\text { ng }\end{array}$ & $860.2 \mathrm{~nm}$ & - & $\begin{array}{l}\text { Hydrophilicity/ phase } \\
\text { separation }\end{array}$ & $\begin{array}{c}\text { Tissue } \\
\text { engineering }\end{array}$ & 106 \\
\hline & PCL & $\begin{array}{c}\text { Glacial } \\
\text { acetic acid }\end{array}$ & $\begin{array}{c}\text { Single nozzle } \\
\text { electrospinni } \\
\text { ng }\end{array}$ & $\begin{array}{c}0.71-1.4 \\
\mu \mathrm{m}\end{array}$ & - & Hydrophilicity & $\begin{array}{c}\text { Tissue } \\
\text { engineering }\end{array}$ & 107-109 \\
\hline & PLGA & THF/DMF & $\begin{array}{c}\text { Single nozzle } \\
\text { electrospinni } \\
\text { ng }\end{array}$ & $\begin{array}{l}200-1000 \\
\mathrm{~nm}\end{array}$ & - & Hydrophilicity & $\begin{array}{c}\text { Tissue } \\
\text { engineering }\end{array}$ & 60,110 \\
\hline & PEO & Water & $\begin{array}{c}\text { Single nozzle } \\
\text { electrospinni } \\
\text { ng }\end{array}$ & $\begin{array}{c}136-148 \\
\mathrm{~nm}\end{array}$ & - & Hydrophilicity & $\begin{array}{c}\text { Drug } \\
\text { delivery }\end{array}$ & 111 \\
\hline & PEO & $\begin{array}{c}\text { Water/ethan } \\
\text { ol }\end{array}$ & $\begin{array}{c}\text { Single nozzle } \\
\text { electrospinni } \\
\text { ng }\end{array}$ & $700 \mathrm{~nm}$ & $\begin{array}{c}\text { Calcinatio } \\
\mathrm{n}\end{array}$ & Nanoporous structure & $\begin{array}{l}\text { Protein } \\
\text { adsorption }\end{array}$ & 112 \\
\hline & PHB & $\begin{array}{l}\text { Chloroform } \\
\text { /dichloroeth } \\
\text { ane }\end{array}$ & $\begin{array}{c}\text { Single nozzle } \\
\text { electrospinni } \\
\text { ng }\end{array}$ & $\begin{array}{c}1.4-1.7 \\
\mu \mathrm{m}\end{array}$ & - & $\begin{array}{l}\text { Hydrophilicity/ } \\
\text { mechanical properties }\end{array}$ & $\begin{array}{l}\text { Wound } \\
\text { dressing }\end{array}$ & 113 \\
\hline
\end{tabular}




\begin{tabular}{|c|c|c|c|c|c|c|c|c|}
\hline & $\mathrm{SnO}_{2}$ & $\begin{array}{l}\text { DMF/ } \\
\text { ethanol }\end{array}$ & $\begin{array}{l}\text { Single nozzle } \\
\text { electrospinni } \\
\text { ng }\end{array}$ & $\begin{array}{c}80-150 \\
\mathrm{~nm}\end{array}$ & $\begin{array}{c}\text { Calcinatio } \\
\mathrm{n}\end{array}$ & Nanoporous structure & Gas sensor & 114 \\
\hline & $\begin{array}{c}\text { PCL/ } \\
\text { gentamic } \\
\text { in/ silver }\end{array}$ & DMSO & $\begin{array}{l}\text { Coaxial } \\
\text { electrospinni } \\
\text { ng }\end{array}$ & - & - & Antibacterial property & $\begin{array}{l}\text { Functional } \\
\text { suture }\end{array}$ & 115 \\
\hline & $\begin{array}{c}\text { PCL/ } \\
\text { platelet } \\
\text { lyophiliz } \\
\text { ate }\end{array}$ & $\begin{array}{l}\text { Chloroform } \\
\text { /ethane }\end{array}$ & $\begin{array}{c}\text { Coaxial } \\
\text { electrospinni } \\
\text { ng }\end{array}$ & $\begin{array}{c}\text { 384-1390 } \\
\mathrm{nm}\end{array}$ & - & Drug release & $\begin{array}{c}\text { Drug } \\
\text { delivery }\end{array}$ & 116 \\
\hline $\begin{array}{l}\text { PCL-Plu } \\
\text { ronic-PC } \\
\quad \mathrm{L}\end{array}$ & - & $\mathrm{DCM} / \mathrm{DMF}$ & $\begin{array}{l}\text { Single nozzle } \\
\text { electrospinni } \\
\text { ng }\end{array}$ & $\begin{array}{c}0.71-3.03 \\
\mu \mathrm{m}\end{array}$ & $\begin{array}{l}\text { Chemical } \\
\text { crosslinki } \\
\text { ng }\end{array}$ & $\begin{array}{c}\text { Hydrophilicity/ } \\
\text { mechanical properties }\end{array}$ & Biomaterial & 117 \\
\hline $\begin{array}{l}\text { PLA-PE } \\
\text { O-PLA }\end{array}$ & $\begin{array}{l}\text { Metronid } \\
\text {-azole }\end{array}$ & $\begin{array}{c}\mathrm{DMF} / \text { aceto } \\
\text { ne }\end{array}$ & $\begin{array}{l}\text { Single nozzle } \\
\text { electrospinni } \\
\text { ng }\end{array}$ & $\begin{array}{c}0.865-2.6 \\
\mu \mathrm{m}\end{array}$ & - & Hydrophilicity & $\begin{array}{c}\text { Tissue } \\
\text { Engineering }\end{array}$ & 118 \\
\hline $\begin{array}{l}\text { PCL- } b \text {-P } \\
\text { SBMA }\end{array}$ & - & DCM & $\begin{array}{l}\text { Single nozzle } \\
\text { electrospinni } \\
\text { ng }\end{array}$ & - & - & Hydrophilicity & Biomaterial & 119 \\
\hline $\begin{array}{l}\text { Silk-elast } \\
\text { in-like } \\
\text { protein }\end{array}$ & - & $\mathrm{H}_{2} \mathrm{O}$ & $\begin{array}{l}\text { Single nozzle } \\
\text { electrospinni } \\
\text { ng }\end{array}$ & $\begin{array}{c}25-1805 \\
\mathrm{~nm}\end{array}$ & - & $\begin{array}{l}\text { Mechanical properties/ } \\
\text { biocompatibility }\end{array}$ & $\begin{array}{c}\text { Tissue } \\
\text { Engineering }\end{array}$ & 120 \\
\hline $\begin{array}{l}\text { PF- } b \text {-PN } \\
\text { IPAM }\end{array}$ & PMMA & THF & $\begin{array}{l}\text { Single nozzle } \\
\text { electrospinni } \\
\text { ng }\end{array}$ & $\sim 500 \mathrm{~nm}$ & - & $\begin{array}{c}\text { Thermal responsivity/ } \\
\text { photoluminescence }\end{array}$ & Sensors & 121 \\
\hline $\begin{array}{l}\text { PF- } b \text {-PN } \\
\text { IPAM- } b \text { - } \\
\text { PNMA }\end{array}$ & PEO & $\mathrm{H}_{2} \mathrm{O}$ & $\begin{array}{l}\text { Single nozzle } \\
\text { electrospinni } \\
\text { ng }\end{array}$ & $\begin{array}{c}358-524 \\
\mathrm{~nm}\end{array}$ & $\begin{array}{c}\text { Chemical } \\
\text { crosslinki } \\
\text { ng }\end{array}$ & $\begin{array}{l}\text { Thermal responsivity/ } \\
\text { photoluminescence }\end{array}$ & Sensors & 122 \\
\hline $\begin{array}{l}\text { PPy- } b \text {-P } \\
\text { NIPAM- } \\
b \text {-PNMA }\end{array}$ & - & Chloroform & $\begin{array}{l}\text { Single nozzle } \\
\text { electrospinni } \\
\text { ng }\end{array}$ & $\begin{array}{c}740-890 \\
\mathrm{~nm}\end{array}$ & $\begin{array}{c}\text { Crosslinki } \\
\mathrm{ng}\end{array}$ & $\begin{array}{c}\text { Thermal } \\
\text { responsivity/metal ion } \\
\text { sensing behaviour }\end{array}$ & $\begin{array}{l}\text { Metal ion } \\
\text { sensor }\end{array}$ & 123 \\
\hline $\begin{array}{c}\text { OT- } b \text {-OP } \\
\text { V }\end{array}$ & PVP & Chloroform & $\begin{array}{l}\text { Coaxial } \\
\text { electrospinni } \\
\text { ng }\end{array}$ & $\begin{array}{c}500-800 \\
\mathrm{~nm}\end{array}$ & $\begin{array}{c}\text { Dip } \\
\text { in ethanol }\end{array}$ & $\begin{array}{l}\text { Morphology/ } \\
\text { self-assembly }\end{array}$ & $\begin{array}{c}\text { Photovoltaic } \\
\text { s }\end{array}$ & 124 \\
\hline $\begin{array}{l}\text { (PFDA-c } \\
\text { o-AA)- } b \text { - } \\
\text { PAN }\end{array}$ & - & DMF & $\begin{array}{l}\text { Single nozzle } \\
\text { electrospinni } \\
\text { ng }\end{array}$ & - & $\begin{array}{c}\text { Thermal } \\
\text { annealing }\end{array}$ & $\begin{array}{c}\text { Morphology/super-hydro } \\
\text { phobicity }\end{array}$ & $\begin{array}{l}\text { Self-cleanin } \\
\text { g material }\end{array}$ & 125 \\
\hline
\end{tabular}

\subsubsection{Biomaterials}

Electrospinning can be used flexibly to fabricate a wide range of BCPs into nanofibres with controlled diameter and porosity, which can be thus designed to mimic the ECM structure. Nonwoven mats with interconnected pores and large surface area-to-volume ratio can provide more structural space for the accommodation of cells, and support essential cell properties, such as migration, proliferation, and differentiation, showing great potential as tissue engineering scaffolds. ${ }^{126,}{ }^{127}$ As discussed throughout this review, nanofibres have a higher surface area per unit volume than bulk materials and coatings, resulting in an increased interface where drug release can occur. ${ }^{128}$ With the addition of BCPs to the electrospinning system, biological applications have been expanded further.

In most cases, the biodegradable polymers are used as scaffolds, where the polymer 
degrades after tissue formation to avoid secondary surgery. Biodegradable functionalization is commonly accomplished by introduction of hydrolytically or enzymatically sensitive groups into the polymer backbone. Electrospun fibrous scaffolds based on biodegradable polymers is one strategy for avoiding postoperative tissue adhesion. ${ }^{88}$ Biodegradation rates must be fine-tuned in respective tissue engineering applications to suit the specific tissue formation and environment. ${ }^{129}$ As such, linear aliphatic polyesters such as PLA, PGA and associated copolymers (PLGA), have been frequently used in tissue engineering and evaluated as skin grafts in vitro.

Molecular design is used to control degradation rates to suit the given application. For example, one such biodegradable copolymer that has been designed for tissue engineering is a multiblock copolymer of poly(ethylene oxide)terephthalate and poly(butylene terephthalate) (PEOT/PBT). The degradation rates could be adjusted by varying the molecular weight and polymer composition..$^{92,93,130}$ As shown in Figure 8, combining electrospinning technology with additive manufacturing, PEOT/PBT multiscale scaffolds can be equipped with mechanical stability and are able to replicate the orientation in collagen fibres, producing a breakthrough solution for critical lesions in the tympanic membrane. ${ }^{131}$ As an emerging biodegradable BCP, triblock copolymer PCEC $^{97-99}$ has aroused great interest. PCL and PEO have been approved by FDA in specific applications such as drug delivery devices, sutures, or adhesion barriers, while PCEC has enhanced hydrophilicity compared to pure PCL. ${ }^{96}$ Curcumin and nano- $\mathrm{Fe}_{3} \mathrm{O}_{4}$ were incorporated into PCEC fibrous skin scaffolds so that the closure of dermal wounds could be accelerated. ${ }^{94,} 95$ In other work, hydroxyapatite has been incorporated within, or deposited onto, PCEC nanofibre membranes to guide bone regeneration in non-load-bearing bone defects. 
a) 3D Fiber Deposition (3DF)

b) Electrospinning (ES)
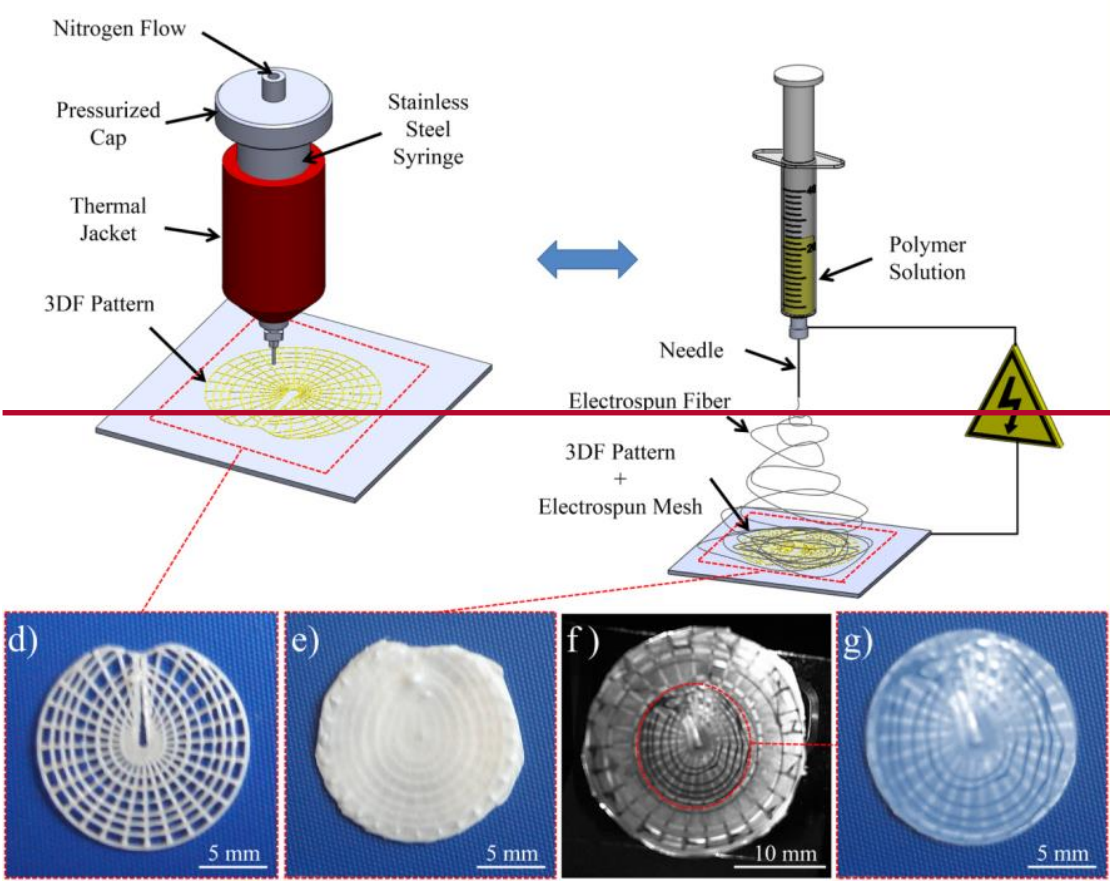

c)

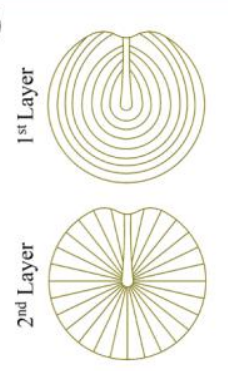

Dual scale TM scaffold

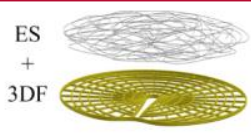

Triple scale TM scaffold

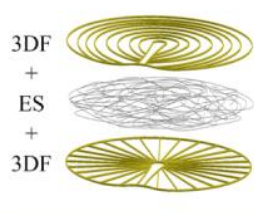

(a) 3D Fiber Deposition (3DF)

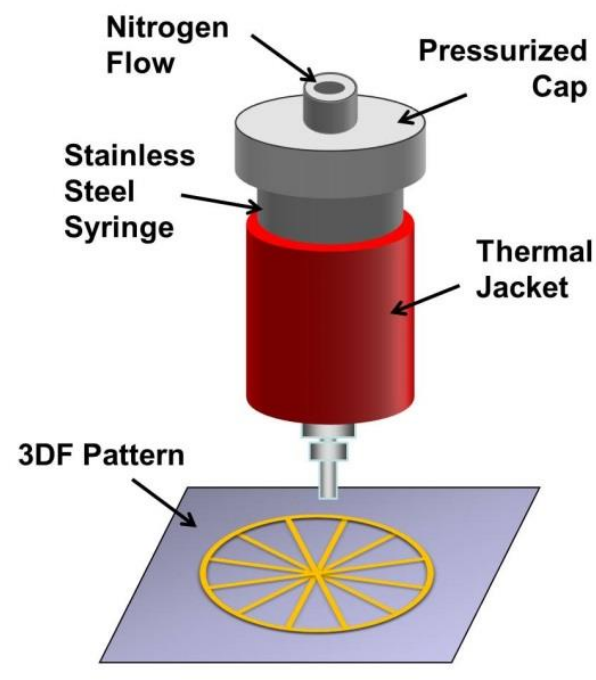

(b) Electrospinning (ES)

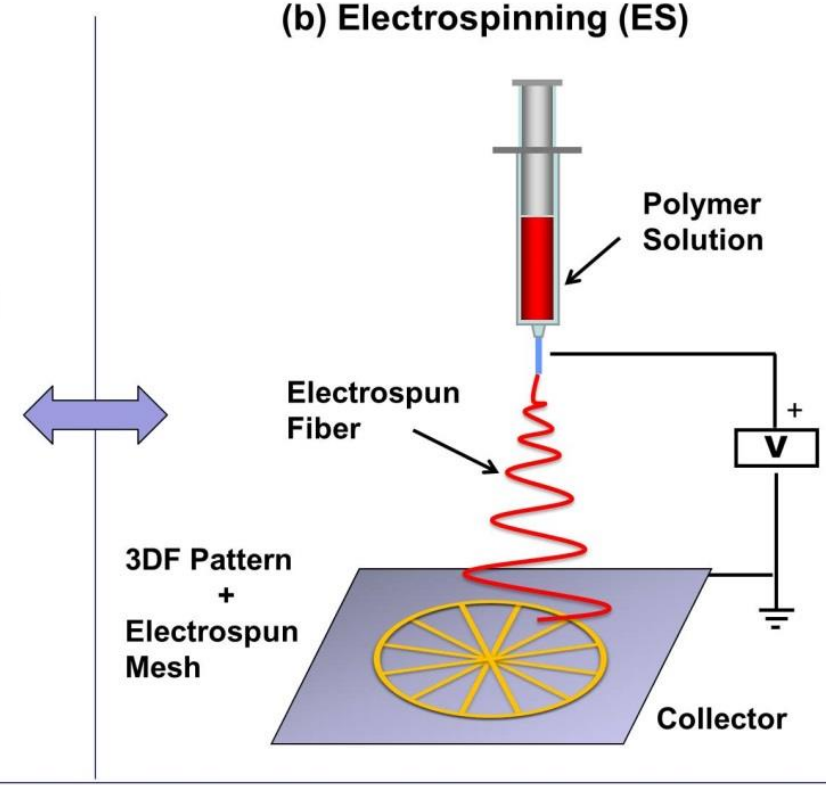

(c)
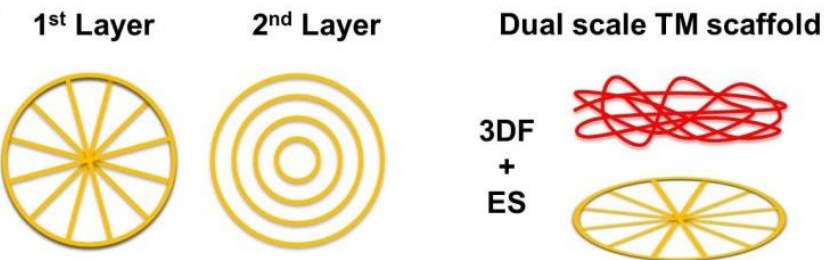

Triple scale TM scaffold

Figure 8. Strategy used for the production of dual and triple scale scaffolds for tympanic membrane scaffolds. (a) The first step involves additive manufacturing the pattern via 3D fibre deposition technique before (b) subsequent coating of the pattern 
with a PEOT/PBT electrospun mesh. (c) Simplified patterns designed with Rhinoceros ${ }^{\circledR}$ with circular concentric microfibres (first layer) and radial microfibres (second layer) and schematic drawings of dual and triple tympanic membrane scaffolds with exploded views. Photographs of the one piece grid layer (d) before and (e) after coating with the electrospun membrane (dual seale tympanic membrane seaffold) and ( $f$ ) a triple seate tympanic membrane scaffeld, as produced on a large frame and $(\mathrm{g})$ the region of interest being carved out, showing circular and radial layers on different membrane sides. Reprinted (adapted) with permission from Mota et al. Biofabrication., 2015, 7, 025005. Copyright (2015) Institute of Physics.- ${ }^{131}$

Although some polymers are biodegradable and generally nontoxic (such as PCL, PLLA, PLCL, silk fibroin and PHB), their use in biomedical applications is primarily limited because of their hydrophobicity, which can prohibit protein adsorption and downstream cell adhesion. Moreover, hydrophobic nanofibrous membranes have been shown to be unsuitable for wound healing. ${ }^{104}$ Water wetting is one of the basic parameters which can be used to characterize the performance of scaffolds. Hydrophilic scaffolds are able to provide an ideal environment for cell proliferation and distribution. When used as a wound dressing, water in contact with hydrophilic fibre surfaces is helpful for preferential deposition of divalent $\mathrm{Ca}^{2+}$ ions at the interface, which leads to rapid initiation of clotting cascades. ${ }^{113}$ When hydrophobic nanofibres are loaded with hydrophilic drug, most of the drug is located near the surface of the nanofibres due to phase separation. This poor interaction between the drug and polymer matrix induces a relatively high initial 'burst' release profile. ${ }^{132}$ Adjusting the hydrophilicity of the nanofibres can control the release profile (through controllable interactions between the drug and the matrix) and thus broaden the application of nanofibres. Blending hydrophilic and hydrophobic homopolymers to improve the hydrophilicity of hydrophobic fibrous membranes is simple (solution blending is performed prior to electrospinning), however the hydrophilic polymer can leach out in water, due to unfavourable interactions between the disparate homopolymers and favourable interactions of the hydrophilic polymer with water, leading to solvation. ${ }^{107}$ 
As ABCs contain both hydrophilic and hydrophobic blocks, they can be used as dual functioning materials in that the hydrophobic segments can anchor to a hydrophobic polymer and the hydrophilic components can assemble on the surface of the fibres to increase water wettability. Many researchers have synthesized ABCs containing PCL or PLLA to overcome issues with hydrophobicity. Hydrophilic segments like $\operatorname{poly}(N$-vinylpyrrolidone) in PVP- $b$-PCL and poly(tetrahydrofuran) in PCL- $b$-PTHF- $b$-PCL, have been shown to self-assemble on the nanofibre surface, which substantially increases hydrophilicity and allows cell attachment and adhesion. ${ }^{102}$ In other work, poly(methyl methacrylate)- $b$-poly(methacrylic acid) (PMMA- $b$-PMAA) was blended with PCL to create composite nanofibres. The nanofibres exhibited a charged surface (due to the carboxylic acid functionality of PMAA), so that chitosan could be deposited on the fibre surface. After chitosan deposition, nanofibrous membranes were produced which possessed both the mechanical resistance of PCL and surface properties of chitosan. ${ }^{101}$ Compared to blending with PEO oligomer, incorporation with higher homogeneity and wettability ABCs was more efficient. ${ }^{133}$ However, it should be noted that excessive hydrophilic character also prohibits cell adhesion due to a thin layer of water that forms over the nonwoven mats, which interferes with cell attachment and adhesion (Figure 9). ${ }^{107,134 \text {, }}$ 135 This highlights the importance of striking the perfect hydrophobic/hydrophilic balance in the system, achieved through molecular design of BCPs, which is made possible by the molecular control delivered by the wide of controlled polymerization techniques available. 


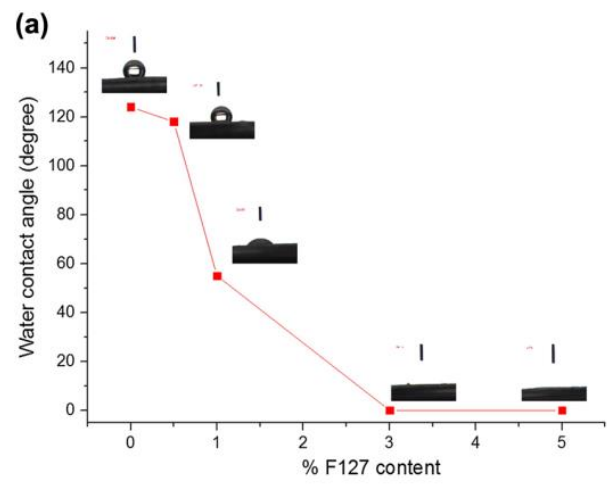

(b)
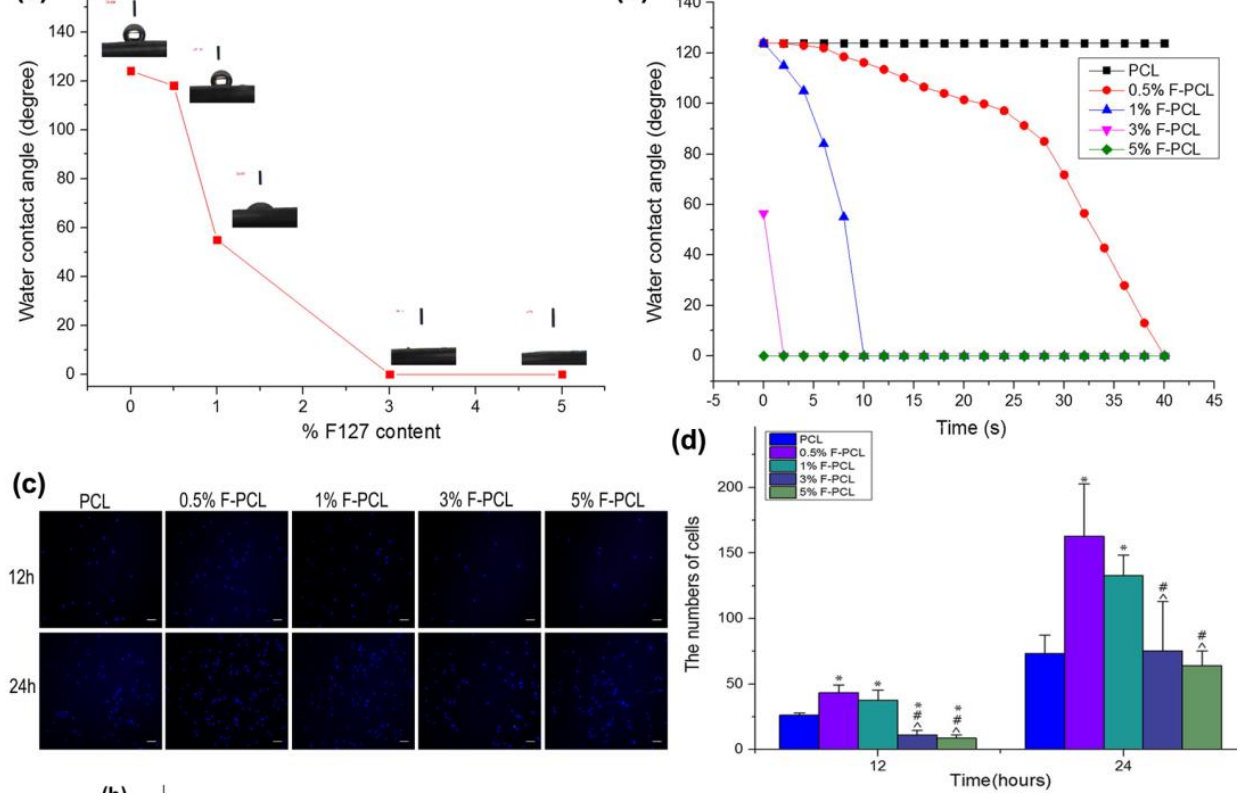

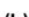

Figure 9. Hydrophilicity and cytocompatibility of PCL/poloxamer (F127) fibre membranes with different F127 contents. (a) Static water contact angle (8 s after deposition); (b) Dynamic water contact angle; (c) DAPI staining of rat bone marrow mesenchymal stem cells (BMSCs) adhered on PCL and PCL/F127 blended scaffolds for 12 and $24 \mathrm{~h}$ (blue color indicates the nucleus, scale bars $=100 \mu \mathrm{m}$ ); and (d) Number of adhered cells on the scaffolds $(*, \wedge$ and \# indicate significant difference from PCL, 0.5\% F-PCL and 1\% F-PCL respectively, $p<0.05) .{ }^{107}$-Images are adapted with permission from Ref. 107. (Copyright 2018 Taylor \& Francis)

Poloxamer (vide supra) is a class of inexpensive commercial ABC which has been incorporated into electrospinning solutions to improve the hydrophilicity of nanofibres. ${ }^{61,108,109,136}$ Control over the length of the polymer blocks allows various customized poloxamers with tunably different properties to be made available. The hydrophobic PPO block of the poloxamer binds to the hydrophobic polymer in the bulk, while the hydrophilic PEO block preferentially assembles as surface brushes or corona, to maximize its interaction with water. ${ }^{106}$ As shown in Figure 10, when acylated poloxamer (A-F127) is blended with SEBS, self-assembly can generate core-shell microfibres with increased poloxamer richness in the shell, as shown by TEM. ${ }^{83}$ In 
addition, poloxamers are thermoresponsive and can induce reversible gelation at low temperature. When poloxamers are used in wound dressings, gelation of poloxamers allows wound dressings to swell and maintain the humidity of the wound, which is important for providing an environment for re-epithelialization and minimizes the chance of infection. ${ }^{104}$ Compared with expensive plasma treated fabrics, this approach delivers PLLA nanofibres with improved surface hydrophilicity and mechanical properties and similar osteogenic differentiation potential. An additional benefit of using poloxamer is its ability to bind lactic acid degradation products (from biodegradable polymers containing polylactide, such as PLLA, PLCL, PGLA), which would otherwise create an acidic microenvironment that prohibits cell regeneration. ${ }^{103}$ During the electrospinning process, poloxamer can act as a surfactant that decreases the surface tension of the solution and effectively suppresses the formation of bead defects, resulting in a smoother fibre morphology. ${ }^{60,105,111,113}$ Finally, blended poloxamer can be selectively removed by dipping in methanol, which etches the nanofibres to increase their roughness, and rougher nanofibres have shown higher serum incorporation. ${ }^{137}$

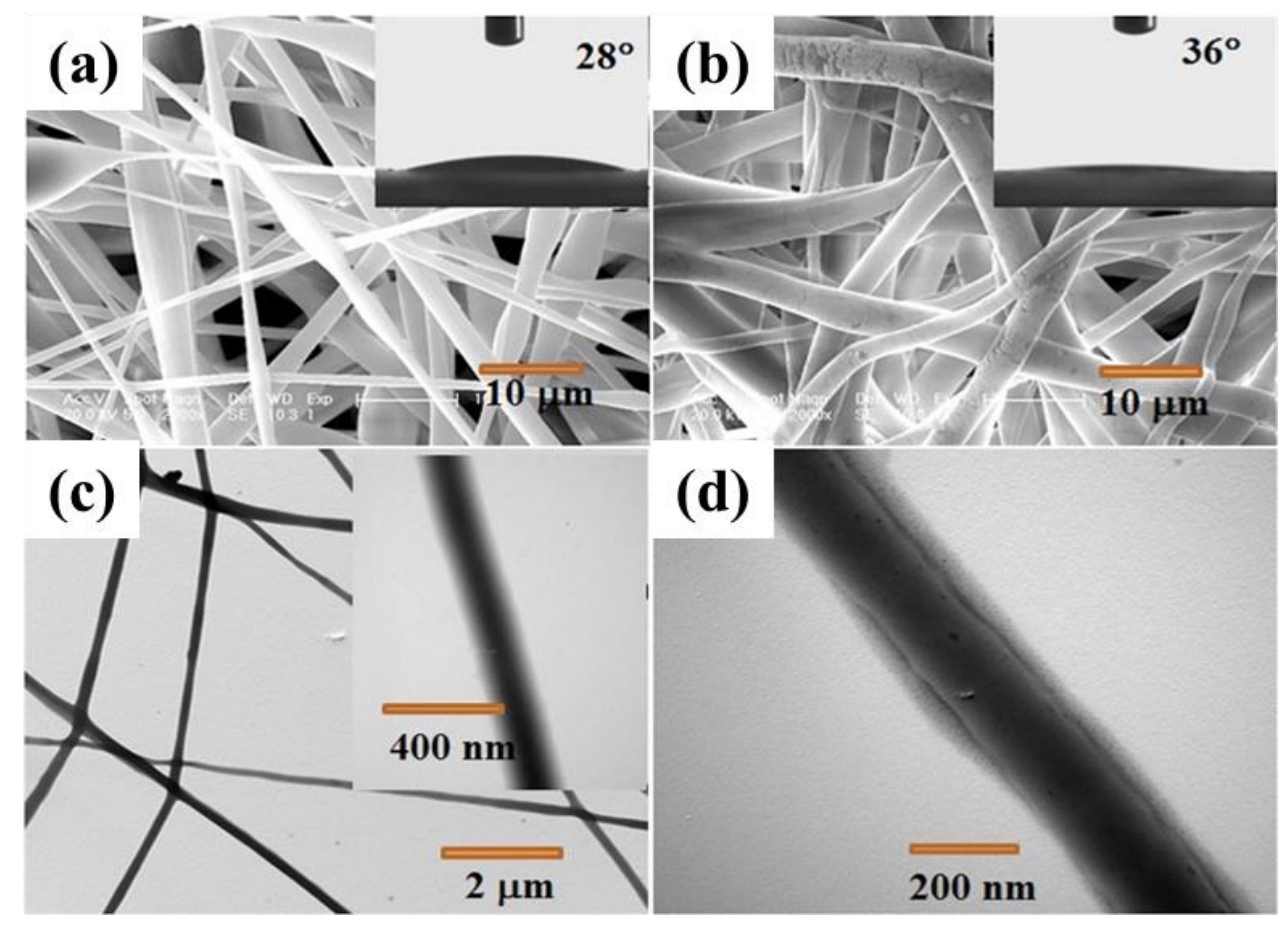

Figure 10. SEM and TEM images of SEBS/acylated poloxamer (A-F127) microfibres. (a) SEM image of electrospun SEBS/A-F127 microfibres; (b) SEM image of electrospun SEBS/A-F127 microfibres after immersion in PBS buffer for $24 \mathrm{~h}$; (c) 
TEM image of SEBS/A-F127/L-ascorbic acid (80/20/20) microfibre; (d) TEM images of microfibres after being exposed to water vapor for $30 \mathrm{~min} .{ }^{83} \underline{\text { Images are adapted }}$ with permission from Ref. 83. (Copyright 2018 Elsevier)

The hydrophilicity of nanofibrous membranes can be improved through the use of novel BCPs without the need for blending with another polymer. For example, PCL- $b$-PEO- $b$-PCL, PCL- $b$-PTHF- $b$-PCL, ${ }^{100}$ PVP- $b$-PCL, ${ }^{102}$ PCL- $b$-PSBMA, ${ }^{119}$ PLA- $b$-PEO- $b$-PLA, ${ }^{118}$ and PCL- $b$-Pluronic- $b$-PCL ${ }^{11738}$ BCPs retain their biocompatible characteristic when an appropriate level of hydrophilicity is attained. Silk-elastin-like protein polymers (SELPs) are genetically-engineered BCPs consisting of silk-like (GAGAGS) and elastin-like (GVGVP) repeating units in defined ratios that have been successfully fabricated into nanofibres. ${ }^{120}$ SELPs have better mechanical properties than silk fibroin (SF) yet retain their inherent biocompatibility. ${ }^{1389-14 \underline{1} \underline{1}}$ Finally, polyisobutylene-based thermoplastic elastomers have also been incorporated into nanofibres for drug delivery and were shown to innovatively achieve slow release of encapsulated drug. ${ }^{128}$

\subsubsection{Separation membranes}

Membrane separation is a technology which selectively fractionates mixtures of materials via pores and/or minute gaps in a continuous structure. Nanofibres can be processed to assemble into a membrane-like web with micrometer-sized pores, which can be directly applied in MF processes to efficiently remove micrometer- or submicrometer-sized particles from water. UF membranes with pore sizes in the range of 1-100 nm can effectively remove colloids, viruses and proteins from mixtures. BCP nanofibres and their blends endow the nonwoven mats with better mechanical performance, hierarchical nanoporous structure and response to external stimuli (e.g., $\mathrm{pH}$, temperature, light, electricity and so on). Thus, these BCP nanofibrous matrices have potential applications for high flux and responsive oil/water separation, ion exchange and large-size protein separation. 
$\mathrm{Li}$ and co-workers ${ }^{69-71}$ have shown a smart fibre membrane by depositing $\mathrm{pH}-$-responsive copolymer fibres on a stainless-steel mesh. PMMA- $b$-P4VP consisting of pH-responsive P4VP and the underwater oleophilic/hydrophilic PMMA endowed the nanofibrous membrane with two switchable wettability states (super-hydrophobicity/superoleophilicity and superhydrophilicity/underwater superoleophobicity). This as-prepared membrane was shown to efficiently separate oil and water using gravity alone through wetting the membrane in acidic water $(\mathrm{pH} 3)$. Importantly, the fibrous membranes were able to maintain their 3D porous surface structure and exhibit excellent switchable wettability after numerous cycles of the separation process (Figure 11). On the basis of using a functional BCP and the porous structure of a nanofibrous surface, a similar block copolymer, PDMS- $b$-P4VP, was used to achieve controllable separation with high flux of oil (hexane) and water though adjustment of $\mathrm{pH}$. By changing the molecular design of the block copolymer, the same group fabricated novel smart membranes based on temperature responsive copolymer PMMA- $b$-PNIPAM. Owing to the thermo-responsive PNIPAM component, the as-prepared membrane exhibited temperature-tuneable oil/water wettability. These smart surfaces with excellent switchable wetting property and separation performance are ideal candidates for oil/water separation. 
a) Water with $\mathrm{pH}=3$

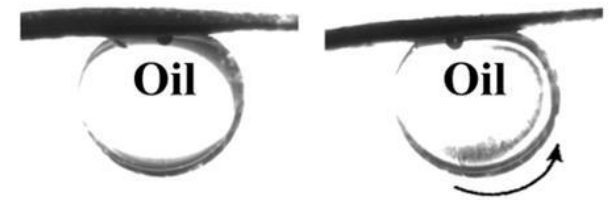

$\mathrm{CA}=152^{\circ}$

$\mathrm{SA}<5^{\circ}$ c) Water with $\mathrm{pH}=3$

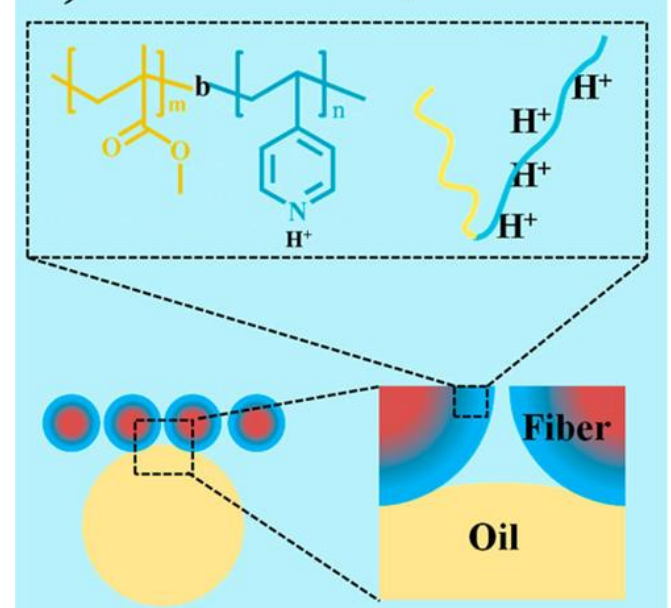

b) Water with $\mathrm{pH}=7$

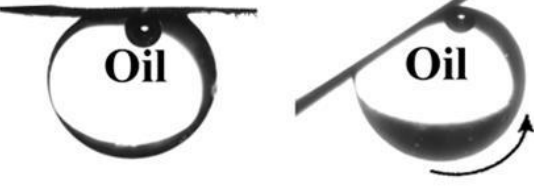

$\mathrm{CA}=146^{\circ}$
$\mathrm{SA}>\mathbf{3 0} 0^{\circ}$ d) Water with $\mathrm{pH}=7$

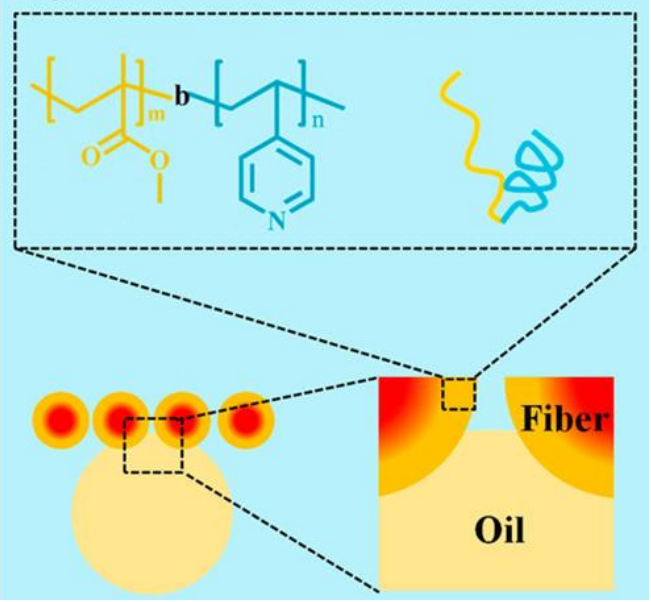

Figure 11. Smart PMMA- $b$-P4VP fibrous membranes fabricated for $\mathrm{pH}$-induced oil/water separation. Oil wettability of the as-prepared fibre membrane in aqueous media with different $\mathrm{pH}$ values: (a) Images of an oil (n-hexane) droplet on the fibre membrane in acidic water ( $\mathrm{pH} 3$ ) with an OCA of $\sim 152^{\circ}$ (left) and a sliding angle of $\sim 4^{\circ}$ (right); (b) Images of an oil (n-hexane) droplet on the as-prepared membrane in neutral water $(\mathrm{pH} 7)$ with an OCA of $\sim 146^{\circ}$ in a horizontal state (left) and a tilted state (right); (c and d) Schematic diagrams of the oil wetting behaviour of the fibre membrane surface in $\mathrm{pH} 3$ and 7 water, respectively. ${ }^{71}$ Images are adapted with permission from Ref. 71. (Copyright 2015 American Chemical Society)

Shi et al. ${ }^{14}$ also fabricated responsive UF membranes by electrospinning amphiphilic PS- $b$-P2VP, where they first investigated the swelling behaviour of the as-spun nanofibres in hot ethanol. The solid nanofibres turned into porous fibres with increased porosity before eventually transforming into isolated spherical micelles with excessive swelling. These membranes with 3D interconnected nanopores can be conveniently modulated by control over the electrospinning duration and show higher 
water permeabilities compared to commercial membranes. However, the fragility of some pure nanofibres has limited their extensive applications. Accordingly, highly flexible and elastic BCPs, consisting of soft and hard segments, can be blended with pure polymer to contribute to the improvement of mechanical (and other) properties. PS and SIS blends processed by electrospinning were further sulfonated with sulfuric acid to produce strong acidic cation IEFs. ${ }^{87}$ Feng et al. prepared submicron IEFs of PS and its blends with SBS. IEFs from PS/SBS fabrics displayed better mechanical performance than those from pure PS (Figure 12) and higher IEC of $4.35 \mathrm{mmol} / \mathrm{g}$ and a copper ion $\left(\mathrm{Cu}^{2+}\right)$ adsorption value of $3.08 \mathrm{mmol} / \mathrm{g} .{ }^{82}$

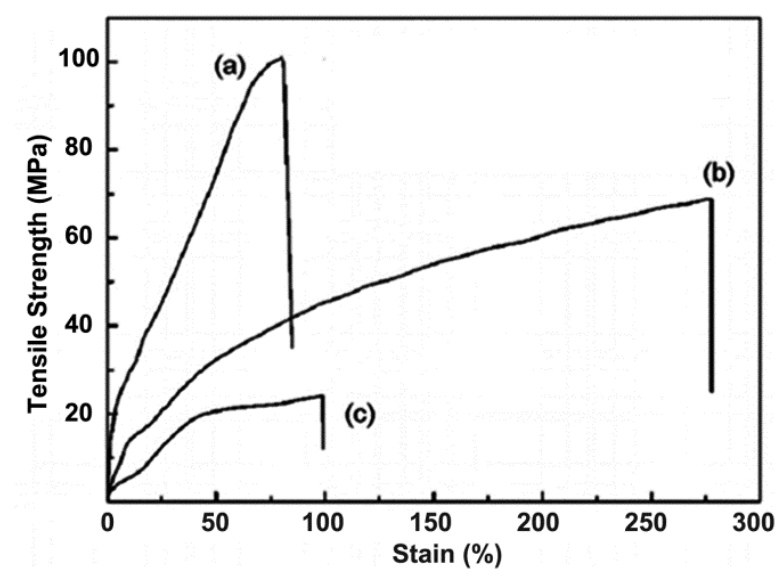

Figure 12. Stress-strain curves of PS and PS/PBS fibrous membranes used for ion-exchange. ${ }^{82}$ (a) Electrospun fibres from pure PS; (b) Electrospun fibres from 80/20 PS/SBS; (c) IEFs from 80/20 PS/SBS.Images are adapted with permission from Ref. 82. (Copyright 2011 Taylor \& Francis)

Besides porous structures built by numerous nanofibres, as discussed throughout this review, nanosized pores in ultrathin fibres can be formed using electrospinning and surfactant-polymer templates, which has shown great potential in protein separation and immobilization. A transparent silica-surfactant composite solution was prepared from silica sol with different concentration of nanostructure-directing agents (nonionic triblock copolymer poloxamer and PEO) before electrospinning. After defined shrinkage and calcination, these electrospun composites yielded corresponding fibres with pores whose diameter ranged from $3.8 \mathrm{~nm}$ to $\sim 450 \mathrm{~nm}$. 
Protein adsorption experiments showed that fibres with $40 \mathrm{~nm}$ diameter pores are favourable for large BSA protein adsorption, where BSA can quickly enter the large pores. The adsorption curves in Figure 13 show that the small-pore fibres exhibit slower BSA adsorption behaviour compared to large-pore fibres. ${ }^{112}$

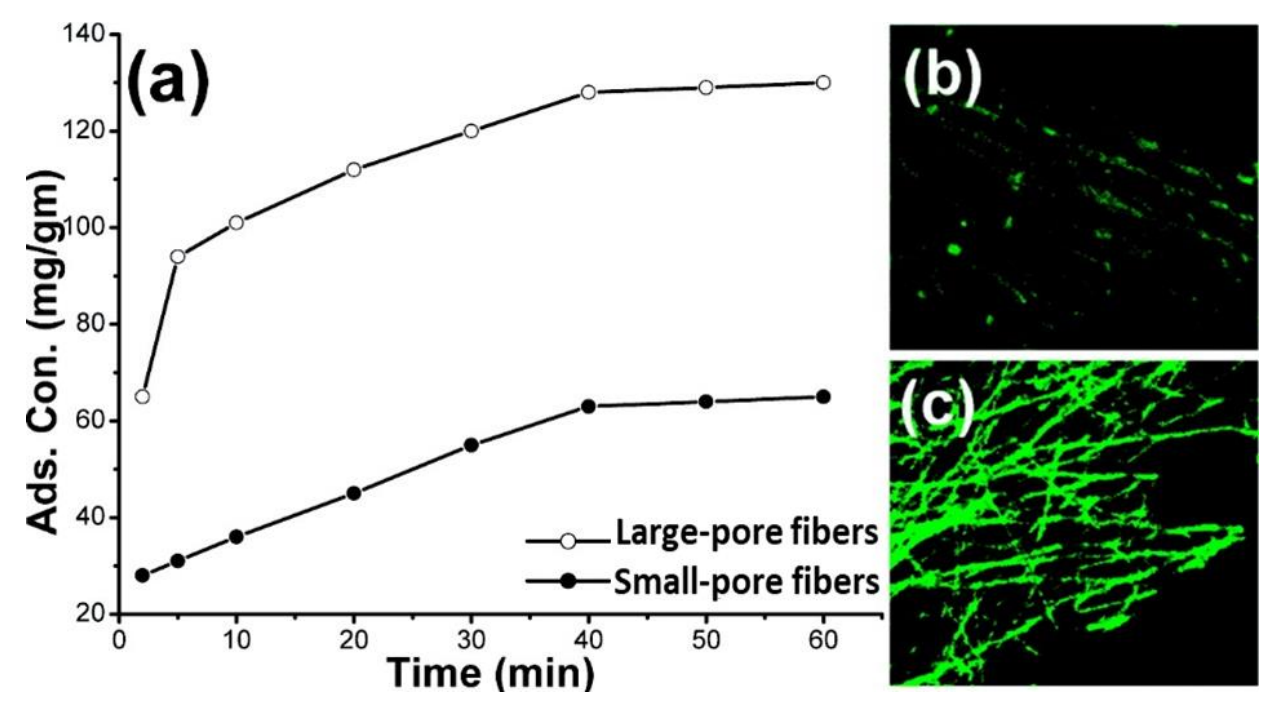

Figure 13. BSA adsorption behaviour of large-pore and small-pore fibres made from P123-PEO complexes. (a) Adsorption rate curves of BSA in $40 \mathrm{~nm}$ (diameter) large-pore fibres and $3.8 \mathrm{~nm}$ small-pore fibres. Fluorescent micrographs of the FITC-BSA-adsorbed small-pore fibres (b) and large-pore fibres (c) following protein adsorption for $1 \mathrm{~h}$. Images are adapted with permission from Ref. 112. (Copyright 2009 American Chemical Society)

\subsubsection{Sensors}

In response to the pressing need for simpler, quicker, highly efficient and smart detection systems, BCP nanofibres containing environmentally responsive segments and fluorescent probes make significant contribution to the development of chemical and biological sensors with superior sensitivity. BCP nanofibre-based sensors have several benefits in sensitivity and specificity over sensors made from other traditional materials. BCPs self-organize towards a stable morphology/structure at thermodynamic equilibrium and when incorporated into nanofibres that have a high surface area-to-volume ratio, this enhances the sensitivity, as well as physical 
properties. Potential applications for these sensors include detection of contaminants in the workplace and the environment, signalling markers in medicine and pathogens in the human body.

BCPs with electronically and optically-sensitive conjugated blocks combined with stimuli-responsive coil blocks are promising sensor candidates. The incorporation of stimuli-responsive blocks has the potential to lead to the variation of photophysical properties for novel sensory devices. Thermoresponsive luminescent nanofibres have been successfully electrospun from conjugated rod-coil PF- $b$-PNIPAM for thermo-tunable colorimetric sensor applications. In order to produce uniform nanofibers, two syringes containing core and shell precursor solutions were utilized in this experiment. High molecular weight PMMA was blended into PF- $b$-PNIPAM to have provide the appropriate rheological properties. The shell THF was injected to retard the rapid solidification and phase separation of the core PF- $b$-PNIPAAM/PMMA blend solution. These BCP nanofibres exhibited reversible photoluminescence spectra-shifting due to the extended/compact structural transformation of the PNIPAM segment leading to the different PF aggregation sizes. Multifunctional BCPs consisting of PNIPAM and $\pi$-conjugated rod blocks undergo a structural variation above the LCST which leads to changes in optoelectronic properties. ${ }^{121}$ PF- $b$-PNIPAM- $b$-PNMA, prepared by ATRP and click coupling, were electrospun into nanofibres (Figure 14). These as-spun nanofibres showed outstanding wettability and reversible on/off transition photoluminescence as the temperature was varied, with high temperature sensitivity and short response times. ${ }^{122}$ 


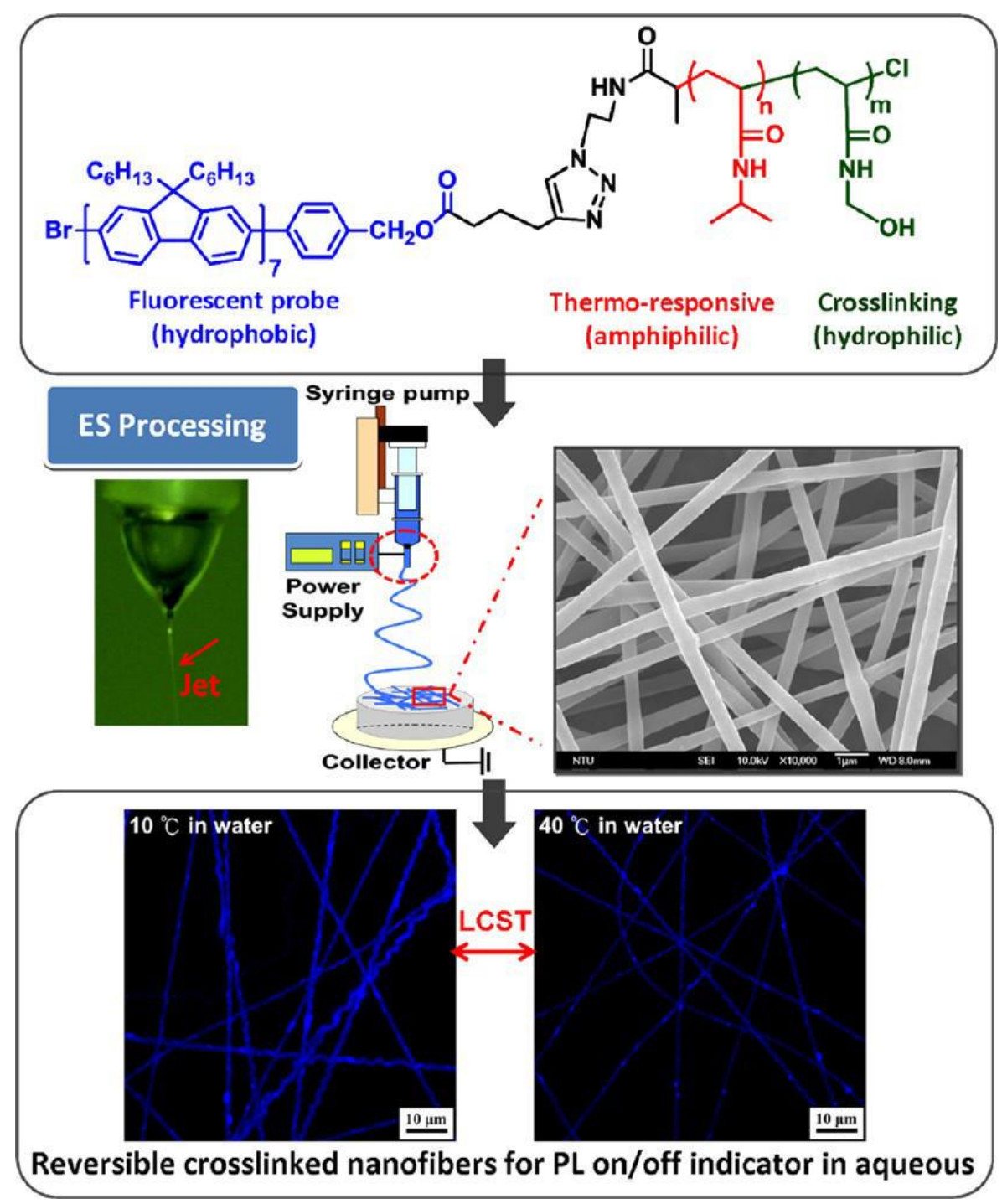

Figure 14. Design of multifunctional sensory electrospinning nanofibres from conjugated rod-coil-coil triblock copolymers of PF- $b$-PNIPAM- $b$-PNMA. ${ }^{122} \underline{\text { Images }}$ are adapted with permission from Ref. 122. (Copyright 2012 American Chemical $\underline{\text { Society) }}$

High sensing performance can also be obtained from 1D nanostructures with higher surface area-to-volume ratio. Soft template poloxamer P123 was added to $\mathrm{SnO}_{2}$ nanofibres to manufacture MOS gas sensors with coarse and loose morphology and high BET surface area. The results of gas sensing measurements indicated that adding P123 improved the sensing performance of $\mathrm{SnO}_{2}$ nanofibres, with increased response time and saturated-detection-concentration, and lower minimum-detection-limit. ${ }^{114}$

Self-assembly of BCPs has been demonstrated as a powerful method to construct 
novel architectures with specific nanofibrous properties. Wang et al. ${ }^{123}$ synthesized similar multifunctional triblock copolymers composed of fluorescent and metal-ion-sensitive PPy, thermoresponsive PNIPAM, and chemically crosslinkable PNMA segments. During thermal crosslinking, the BCP nanofibres concomitantly self-assembled to form nanoscale spherical aggregates with crosslinked PNMA located at the core, PNIPAM in the central layer, and PPy as the shell. Detectable changes in photoluminescence were recorded at different temperatures or different $\mathrm{Fe}^{3+}$ ion concentrations. The higher sensing ability toward subtle changes in external stimuli render the nanofibres suitable for metal ion sensory device applications. At the same time, smart nanofibres have also been developed as sensors to detect chemical vapour.

Gao et al. ${ }^{84}$ prepared superhydrophobic and electrically conductive nanofibrous membranes which were mechanically robust, anti-corrosive, and sensitive to both polar and nonpolar chemical vapours. The membranes were composed of blends of carbon nanofibres (CNFs)/PU/SEBS, where PU is swollen by polar solvents, such as THF and acetone, while SEBS is sensitive to nonpolar solvents. More specifically, the polystyrene segment in SEBS detects the more aromatic solvent vapour (e.g. benzylics), whereas the poly(ethylene-co-butylene) segment is responsible for the detection of more aliphatic vapours. Additionally, when used as a chemical vapour sensor, the nanofibre composites displayed excellent recyclability (shown in Figure 15). 

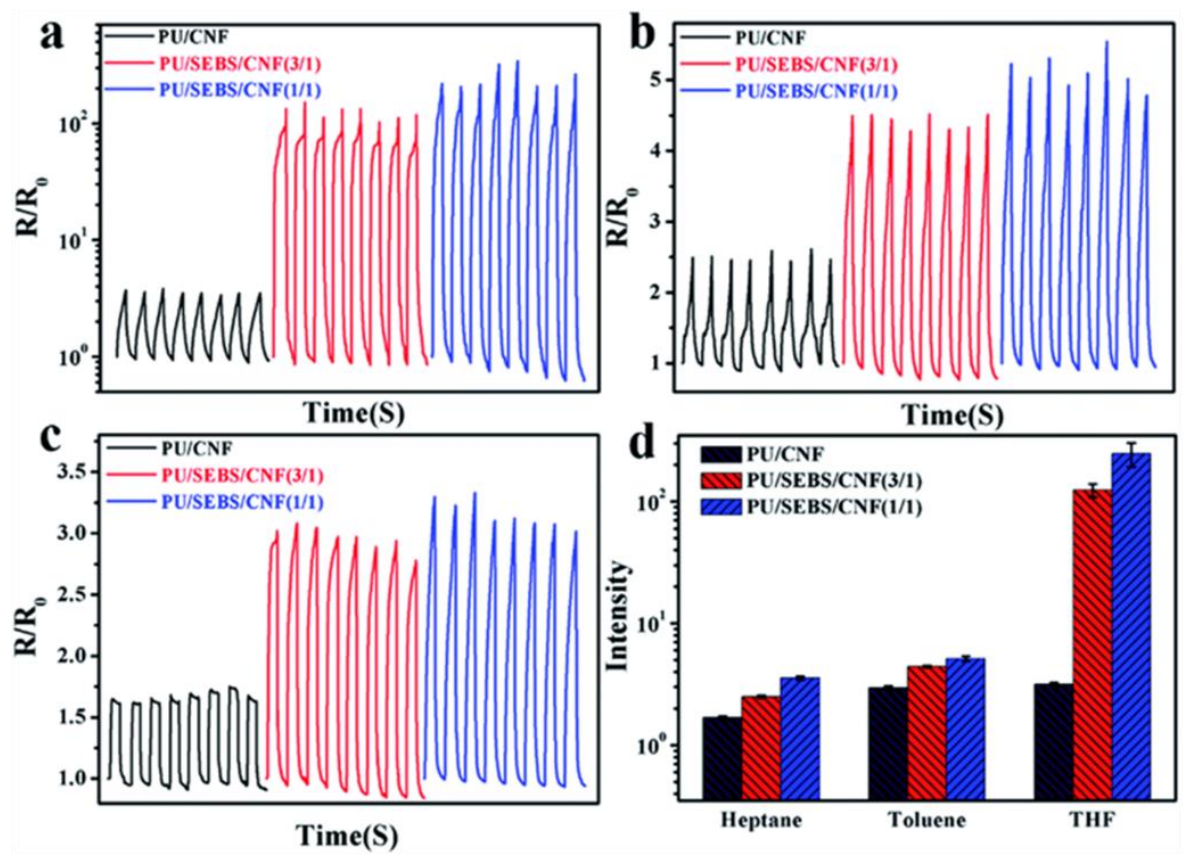

Figure 15. Cyclic vapor (a) THF, (b) toluene and (c) heptane sensing behaviour of PU/SEBS/CNF composites with different SEBS contents. Each cycle of the vapor sensing includes $1 \mathrm{~min}$ of vapour adsorption $\left(\mathrm{R} / \mathrm{R}_{0}\right.$ increase) and $1 \mathrm{~min}$ of de-adsorption of the vapour $\left(\mathrm{R} / \mathrm{R}_{0}\right.$ decrease). (d) Summary of the responsivity $(\mathrm{RCI})$ of the nanofibre composite towards the three different solvent vapors. ${ }^{-84} \underline{\text { Images are }}$ adapted with permission from Ref. 84. (Copyright 2018 Royal Society of Chemistry)

When nanofibres are used to construct biosensors, the surface functionality, hydrophilicity and water solubility of nanofibres must be taken into consideration. Frey et $a l .{ }^{89}$ created biotin surface-functionalized hydrophilic non-water-soluble biocompatible PLA nanofibres together with PLA- $b$-PEO block polymers. They found that the incorporation of PLA- $b$-PEO not only decreased the diameter of the fibres and increased their wettability, but also aided the migration of biotin to the surface by producing a 506\% increase of surface-available biotin. The effects of both solvent and copolymer block length on the stability of PLA/PLA- $b$-PEO and PLA/PLA- $b$-PEO-biotin fibres in water was investigated..$^{90}$ 


\subsubsection{Electronic materials}

Nowadays, electronic materials are being used widely as core elements in a variety of device applications in daily life. The development of flexible energy storage devices, such as lithium-ion batteries and wearable electronic fabrics, has attracted significant attention. To integrate highly efficient organic solar cells into wearable fabrics, Kalra's group ${ }^{124}$ reported the fabrication of electrospun nanofibres of a fully conjugated rod-rod OT- $b$-OPV to revolutionize wearable photovoltaics. Coaxial electrospinning was performed to construct coaxial nanofibres with a sacrificial PVP shell and an OT- $b$-OPV core due to both the low molecular weight and rigid rod conformation of both blocks of OT- $b$-OPV. The PVP was then selectively removed to form pure OT- $b$-OPV nanofibres. X-ray scattering was used to quantify the molecular ordering within the individual blocks (Figure 16). The periodic repeat units of the nanofibres based on OT (electron donor material) and OPV (electron acceptor material) with characteristic spacing of less than the exciton diffusion distance $(\sim 10$ $\mathrm{nm})^{1412}$ was expected to facilitate dissociation of electrons and holes to enhance the efficiency of organic solar cells.

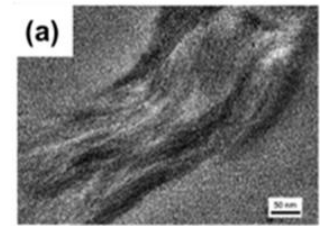

(c)

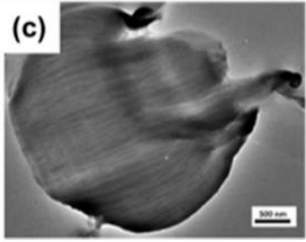

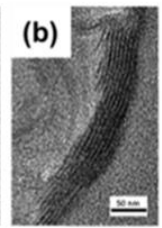

(d)

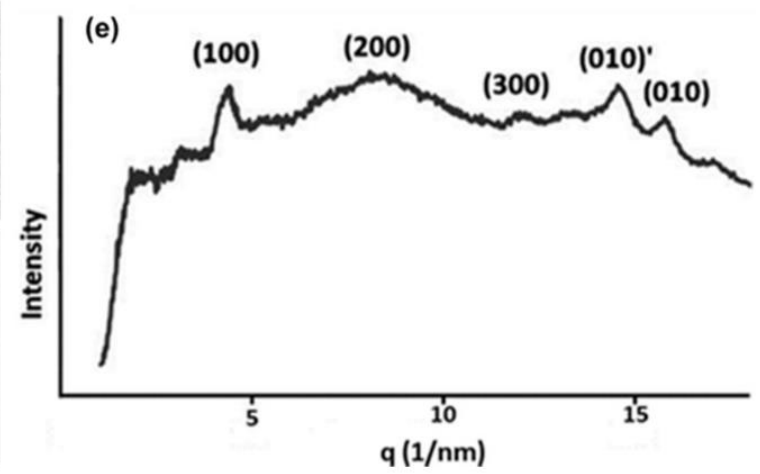

Figure 16. Self-assembled structures of conjugated OT- $b$-OPV rod-rod oligomeric nanofibres. (a-d) TEM micrographs of microtomed nanofibres along and across the fibre axis; and (e) WAXS data of the as-prepared nanofibres. ${ }^{124}$ Images are adapted with permission from Ref. 124. (Copyright 2013 Royal Society of Chemistry)

As electrode materials in $\mathrm{Li}$-ion batteries, novel cigar-like $\mathrm{TiO}_{2}$ nanofibres (as 
discussed previously, see Figure 6) with an outer-shell and inner-continuous-pore structure, fabricated by $\mathrm{Li}$ and co-workers, exhibited much higher charge/discharge capacity and cyclic stability (Figure 17). ${ }^{73}$ They coupled the self-assembly of PS- $b$-PEO containing titanium precursors (bottom-up) with electrospinning (top-down) to construct the hierarchically porous structures. Compared with commercial nanoparticles (P25), these nanofibres exhibited improved mechanical properties and enhanced performance in Li-ion batteries.
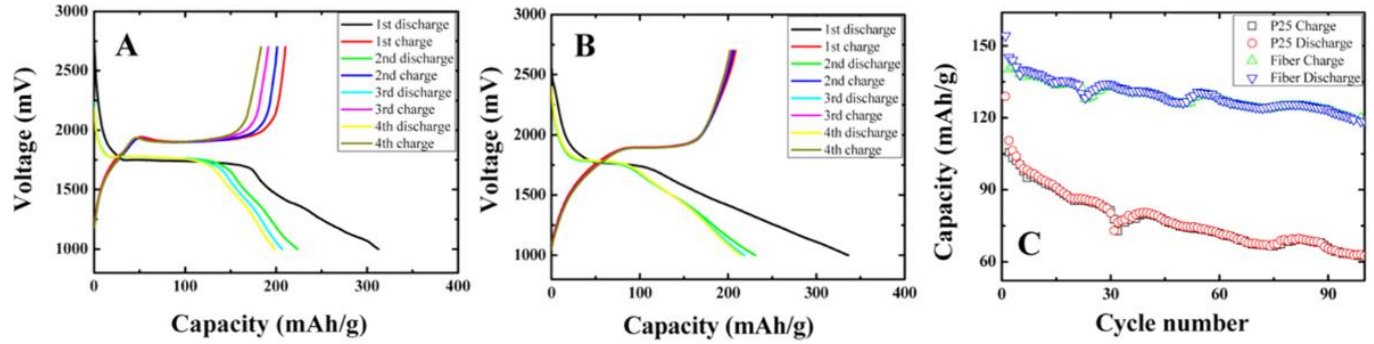

Figure 17. Novel cigar-like nanofibres with an outer-shell and inner-continuous-pore structure were electrospun from PS- $b$-PEO and titanium precursors. Early stage of charge/discharge of (a) commercial nanoparticles, P25; (b) nonwoven fabrics from cigar-like $\mathrm{TiO}_{2}$ fibres and (c) discharge capacity as a function of cycle number. Images are adapted with permission from Ref. 73. (Copyright 2013 American Chemical Society)

Bryan et al..$^{75}$ also reported a simple method of fabricating hierarchically structured $\mathrm{Ni} / \mathrm{NiO} / \mathrm{MnO}_{\mathrm{x}} /$ carbon fibre composites based on nickel and manganese nitrate salts, citric acid, phenolic resin, and an amphiphilic block copolymer via electrospinning and carbonization for lithium ion battery anodes. They used a precursor to improve the stability of the nanostructures on transformation to metal oxide and included carbon precursor (resol) in the spinning solution to limit the growth of any metal oxide crystals. Additionally, PMPEOMA- $b$-PBA was utilized as a template to introduce porosity within the nanofibres, enhance electrolyte diffusion and accommodate strain associated with lithiation/delithiation of the final product. The importance of electronic conductivity and continuous charge-transfer pathways for high performance lithium ion battery anodes was illustrated in this work. 


\section{5 $\underline{3.5}$ Other applications}

In addition to their use as biomaterials, separation membranes, sensors and electronic materials, other applications of note include self-cleaning materials and catalysis, among others. Generally, the wetting behaviour of a solid surface is important for various commercial applications. Self-cleaning materials have a surface which is superhydrophobic with static water contact angles greater than $150^{\circ}$ and a low rolling angle. It has been recognized that the surface morphology/microstructure and low surface free energy are the two most important factors in obtaining superhydrophobicity. Micro- and nanostructuration of the diblock copolymer P(FDA-co-AA)- $b$-PAN coating was obtained by electrospinning. They electrospun nanofibres of the tailored diblock perfluoropolymer on aluminium surfaces to endow the surface with durable superhydrophobicity and improved corrosion resistance. ${ }^{125}$

However, unstable superhydrophobicity is an inherent problem that restricts the practical application of superhydrophobic materials. Wang's group ${ }^{85}$ firstly produced more uniform, intact beads by electrospinning self-assembled BCP solutions. Then they further combined electrospinning, electrospraying and thermal annealing techniques to fabricate rinse-resistant superhydrophobic fabrics with controlled microand nanostructures. SEBS was first electrospun to fabricate fibres as a base supporting mesh and SEBS beads were electrosprayed onto the fibrous SEBS mesh by careful design of a selective co-solvent system. The co-solvent system induced microphase separation of the block copolymer in solution prior to processing, which reduced chain entanglement in the polymer solution resulting in the production of robust microbeads (rather than nanofibres). The introduction of beads onto the nanofibrous bed successfully increased the static water contact angle to $156^{\circ}\left( \pm 1^{\circ}\right)$, while the sliding/rolling angle decreased to $8^{\circ}\left( \pm 1^{\circ}\right)$. In order to increase the stability of the intricate nano- and microstructure of the structurally composite material, thermal annealing was utilized to induce self-assembly and entanglement of molecular chains 
binding the fibres and beads together (Figure 18). The superhydrophobicity of the composite membrane were well retained and stable due to its locked hierarchical surface structure. This example also highlights the important of solvent selection/design when electrospinning (or electrospraying) and provides further control over morphology (and therefore properties). ${ }^{86}$

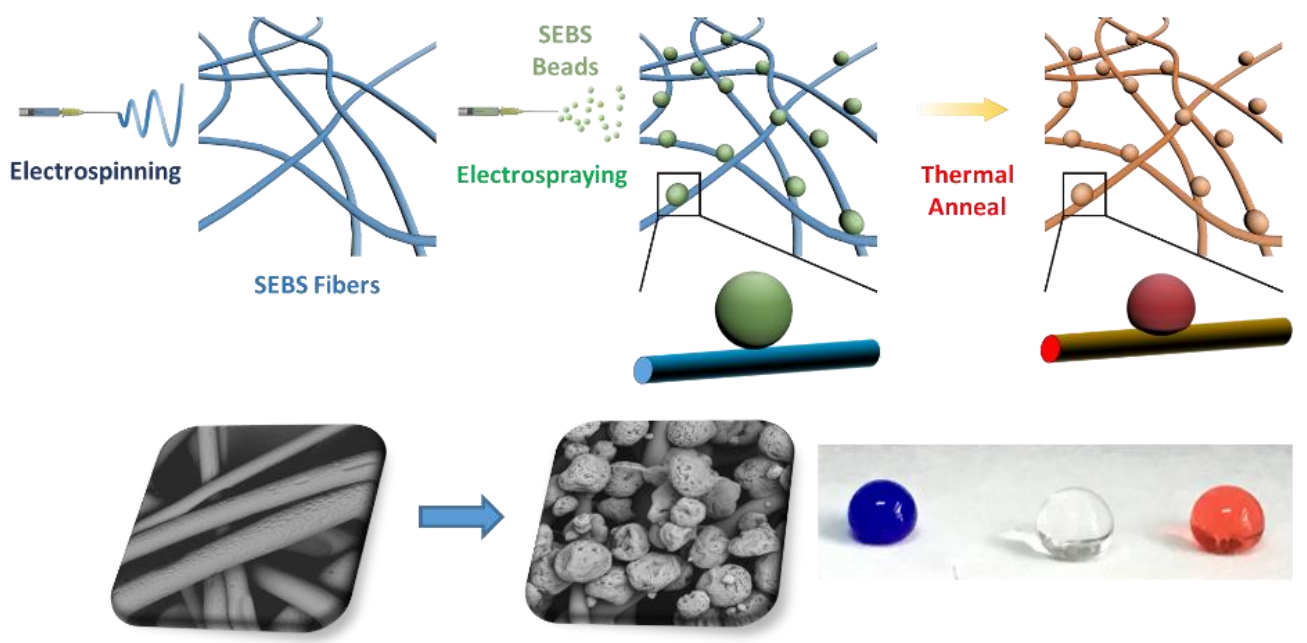

Figure 18. The fabrication process of rinse-resistant superhydrophobic fabrics from electrospraying SEBS beads on electrospun SEBS fibres. ${ }^{86}$ Images are adapted with permission from Ref. 86. (Copyright 2017 Elsevier)

$\mathrm{Li}$ and co-workers ${ }^{74}$ fabricated the same outer-shell and inner continuous-pore structure as previously discussed. They further investigated how to control the structure in detail and studied the photocatalytic activity of the $\mathrm{TiO}_{2}$ fabrics by taking the photodegradation of $\mathrm{RhB}$ as an example. Figure 19 shows the photocatalytic degradation efficiency of $\mathrm{TiO}_{2}$ fabrics prepared using PS-PEO with different block ratios, indicating that the nonwoven fabrics with "double fibres" exhibit excellent photocatalytic activity. 

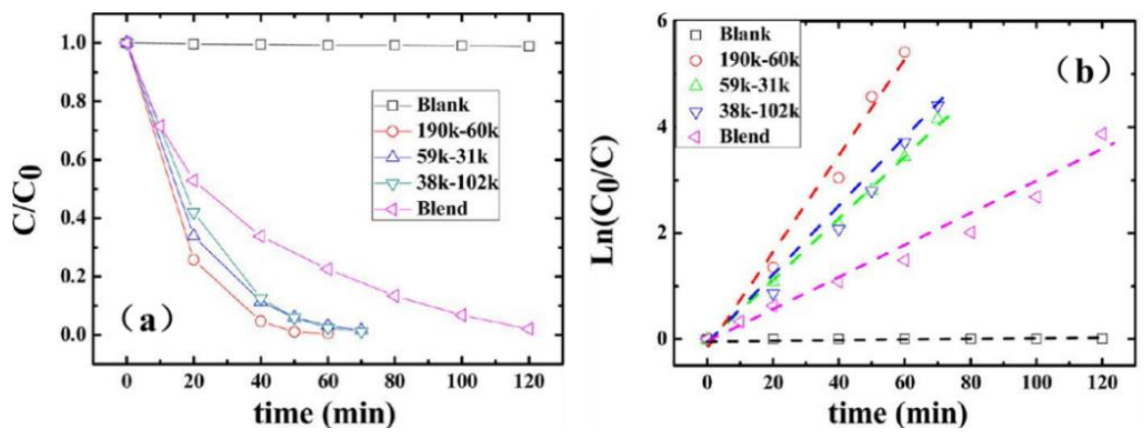

Figure 19. Photocatalytic behaviours of $\mathrm{TiO}_{2}$ fabrics: (a) Photocatalytic degradation of $\mathrm{RhB}$ on as-prepared samples with different block ratios between PS and PEO; (b) Apparent first order plots, where $\ln \left(\mathrm{C}_{0} / \mathrm{C}\right)=\mathrm{kt}$ of $\mathrm{RhB}$ degradation kinetic plots; $\mathrm{C}_{0}$ and $\mathrm{C}$ are the initial $\mathrm{RhB}$ concentration and concentration at irradiation time (min), respectively. Images are adapted with permission from Ref. 74. (Copyright 2016 American Chemical Society)

\section{Conclusions}

Electrospinning can be used to fabricate fibrous nanomaterials with a high surface area-to-volume ratio from a broad spectrum of polymers. The composition, porosity, morphology, and alignment of nanofibres can be adjusted by a variety of strategies. In parallel, BCPs are generally used in the solid state to create nanostructural patterns with control over size and shape due to their self-assembling behaviour towards thermodynamic equilibrium. BCP nanofibres combine the advantages of electrospun materials with those of BCPs. Excellent control over bulk properties (e.g., mechanical performance, conductivity, magnetism), surface properties (e.g., superhydrophobicity, amphiphilicity, coarse structure), biodegradation, mesoporosity and microphase separation make BCP nanofibres stand out. Most research in this area has focused on the fabrication of nanofibrous BCP materials for their application as tissue engineering scaffolds, drug delivery devices, wound dressings, oil/water separation membranes, ion-exchange membranes, protein adsorption materials, gas sensors, biosensors and electronic materials. As biomaterials, on the basis of retaining the advantages of the original materials, the properties of BCP nanofibres were improved, in particular the mechanical strength, controllable hydrophilic-hydrophobic surface 
energy, and controllable biodegradation rate. Notably, BCP nanofibres have been applied in the field of medicine due to the efficient drug carrying ability and sustained (and controllable) release performance. For separation membranes and sensors, the functional segments of BCPs and the tuneable porosity of nonwoven mats play significant roles. Nanofibres composed of stimuli-responsive blocks are capable of altering their chemical and/or physical properties upon exposure to external stimuli so as to be recognized as "smart" materials. The range of hierarchical structures and controlled organization/patterning within nanofibres and composites have been thoroughly investigated and discussed herein. This review also highlights the importance of processing parameters when exploiting BCPs in electrospinning. As more novel BCPs are synthesized, and electrospinning techniques are developed further, future advances of related science and technology will also likely be driven by these applications. 


\section{Acknowledgements}

The authors thank the financial support from National Key R\&D Program of China (No. 2017YFC11050003), National Natural Science Foundation of China (Nos. 21404061, 21807046), Guangdong Innovative and Entrepreneurial Research Team Program (No. 2016ZT06C322), the Fundamental Research Funds for the Central Universities (2018PY05), and Scientific Research Foundation of Xiangnan University for High-Level Talents. PDT thanks the State Administration for Foreign Experts Affairs (SAFEA) and the Royal Society of Chemistry for a Visiting Researcher Program grant to China.

\section{References}

1. S. E. Stein, S. R. Heller and D. Tchekhovski, The IUPAC Chemical Identifier, 2003, 131-143.

2. A. N. Moore and J. D. Hartgerink, Acc. Chem. Res., 2017, 50, 714-722.

3. Y. Wu, C. Chen, Y. Jia, J. Wu, Y. Huang and L. Wang, Appl. Energ., 2018, 210, 167-181.

4. H. R. Pant, M. P. Bajgai, K. T. Nam, Y. A. Seo, D. R. Pandeya, S. T. Hong and H. Y. Kim, J. Hazard. Mater., 2011, 185, 124-130.

5. J. M. Deitzel, J. Kleinmeyer, D. Harris and N. C. B. Tan, Polymer, 2001, 42, 261-272.

6. N. Bhardwaj and S. C. Kundu, Biotechnol. Adv., 2010, 28, 325-347.

7. F. E. Ahmed, B. S. Lalia and R. Hashaikeh, Desalination, 2015, 356, 15-30.

8. S. Agarwal, A. Greiner and J. H. Wendorff, Prog. Polym. Sci., 2013, 38, 963-991.

9. A. Greiner and J. H. Wendorff, Angew. Chem. Int. Ed. Engl., 2007, 46, 
$5670-5703$.

10. J. Xue, J. Xie, W. Liu and Y. Xia, Acc. Chem. Res., 2017, 50, 1976-1987.

11. N. E. Zander, Polymers, 2013, 5, 19-44.

12. T. Abudula, U. Saeed, N. Salah, A. Memic and H. Al-Turaif, J. Nanosci. Nanotechno., 2018, 18, 8240-8251.

13. W.-T. Koo, S.-J. Choi, S.-J. Kim, J.-S. Jang, H. L. Tuller and I.-D. Kim, J. Am. Chem. Soc., 2016, 138, 13431-13437.

14. X. Shi, Z. Xu, C. Huang, Y. Wang and Z. Cui, Macromolecules, 2018, 51, 2283-2292; $\quad$ http://www.safecarecampaign.org/uti.html; $\quad$ http://www. hitachi-chem.co.jp; $\quad$ https://mamanam.com; http://www.elektryk24h.info; https://www.demilked.com.

15. G.-Z. Yang, J.-J. Li, D.-G. Yu, M.-F. He, J.-H. Yang and G. R. Williams, Acta. Biomater., 2017, 53, 233-241.

16. J. Cheng, Y. Jun, J. Qin and S.-H. Lee, Biomaterials, 2017, 114, 121-143.

17. J. I. Kim and C. S. Kim, Mat. Sci. Eng. C. Mater., 2018, 91, 824-837.

18. L. Ghasemi-Mobarakeh, M. P. Prabhakaran, M. Morshed, M.-H. Nasr-Esfahani and S. Ramakrishna, Biomaterials, 2008, 29, 4532-4539.

19. E. Ko, J. S. Lee, H. Kim, S. Y. Yang, D. Yang, K. Yang, J. Lee, J. Shin, H. S. Yang, W. Ryu and S.-W. Cho, ACS Appl. Mater. Inter., 2018, 10, 7614-7625.

20. N. Tuancharoensri, G. M. Ross, S. Mahasaranon, P. D. Topham and S. Ross, Polym. Int., 2017, 66, 1463-1472.

21. Q. Yao, J. G. L. Cosme, T. Xu, J. M. Miszuk, P. H. S. Picciani, H. Fong and H. Sun, Biomaterials, 2017, 115, 115-127.

22. E. R. Kenawy, G. L. Bowlin, K. Mansfield, J. Layman, D. G. Simpson, E. H. Sanders and G. E. Wnek, J. Control Release., 2002, 81, 57-64.

23. L. G. Wang, M. Wang, P. D. Topham and Y. Huang, Rsc Advances, 2012, 2, $2433-2438$.

24. Y. Li, B. Zhou, G. Zheng, X. Liu, T. Li, C. Yan, C. Cheng, K. Dai, C. Liu, C. Shen and Z. Guo, J. Mater. Chem. C., 2018, 6, 2258-2269.

25. C. L. Casper, J. S. Stephens, N. G. Tassi, D. B. Chase and J. F. Rabolt, 
Macromolecules, 2004, 37, 573-578.

26. A. Isakova, O. Efremova, N. Pullan, L. Lueer and P. D. Topham, Rsc Advances, 2016, 6, 6598-6606.

27. C. W. Pester, C. Liedel, M. Ruppel and A. Böker, Prog. Polym. Sci., 2017, 64, $182-214$.

28. F. H. Schacher, P. A. Rupar and I. Manners, Angew. Chem. Int. Ed. Engl., 2012, 51, 7898-7921.

29. J. Shin, Y. W. Kim, and G. J. Kim, Appl. Chem. Eng, 2014, 25, 121-133.

30. M. A. Hillmyer and W. B. Tolman, Acc. Chem. Res., 2014, 47, 2390-2396.

31. D. A. Olson, L. Chen and M. A. Hillmyer, Chem. Mater., 2008, 20, 869-890.

32. P. Theato, B. S. Sumerlin, R. K. O'Reilly and T. H. Epps, III, Chem. Soc. Rev., 2013, 42, 7055-7056.

33. I. Cobo, M. Li, B. S. Sumerlin and S. Perrier, Nat. Mater, 2015, 14, 143-159.

34. P. D. Topham, J. R. Howse, C. M. Fernyhough and A. J. Ryan, Soft. Matter, 2007, 3, 1506-1512.

35. A. Blanazs, S. P. Armes and A. J. Ryan, Macromol. Rapid Commun., 2009, 30, 267-277.

36. M. Elsabahy and K. L. Wooley, J. Polym. Sci., Part A: Polym.Chem., 2012, 50, $1869-1880$

37. J. Y. Cheng, A. M. Mayes and C. A. Ross, Nat. Mater, 2004, 3, 823-828.

38. H. A. Klok and S. Lecommandoux, Adv. Mater., 2001, 13, 1217-1229.

39. A. V. Ruzette and L. Leibler, Nat. Mater, 2005, 4, 19-31.

40. Y. Mai and A. Eisenberg, Chem. Soc. Rev., 2012, 41, 5969-5985.

41. I. W. Hamley, Nanotechnology, 2003, 14, R39-R54.

42. H. Erothu, J. Kolomanska, P. Johnston, S. Schumann, D. Deribew, D. T. W. Toolan, A. Gregori, C. Dagronlartigau, G. Portale and W. Bras, Macromolecules, 2015, 48, 2107-2117.

43. I. Botiz and S. B. Darling, Mater. Today., 2010, 13, 42-51.

44. S. B. Darling, Prog. Polym. Sci., 2007, 32, 1152-1204.

45. M. W. Matsen and M. Schick, Curr. Opin. Colloid. In., 1996, 1, 329-336. 
46. M. A. Morris, Microelectron. Eng., 2015, 132, 207-217.

47. Y. Zhu, B. Yang, S. Chen and J. Du, Prog. Polym. Sci., 2017, 64, 1-22.

48. H. Fong and D. H. Reneker, J. Polym. Sci. Pol. Phys., 1999, 37, 3488-3493.

49. S. Ribeiro, P. Costa, C. Ribeiro, V. Sencadas, G. Botelho and S. Lanceros-Méndez, Comopos Part B-Eng., 2014, 67, 30-38.

50. L. Fan, Y. Xu, X. Zhou, F. Chen and Q. Fu, Polymer, 2018, 153, 61-69.

51. S. Q. Feng, X. Y. Shen, Z. Y. Fu and Y. L. Ji, J. Appl. Polym. Sci., 2009, 114, $1580-1586$.

52. S. Chuangchote, A. Sirivat and P. Supaphol, Polym. J., 2006, 38, 961-969.

53. W. Rungswang, M. Kotaki, T. Shimojima, G. Kimura, S. Sakurai and S. Chirachanchai, Polymer, 2011, 52, 844-853.

54. V. Kalra, P. A. Kakad, S. Mendez, T. Ivannikov, M. Kamperman and Y. L. Joo, Macromolecules, 2006, 39, 5453-5457.

55. Z. Zhou, K. Cao, X. Chen, M. Nguyen, S. Talley, R. B. Moore, S. M. Martin and G. Liu, Mol. Syst. Des. Eng., 2018, 3, 357-363.

56. M. L. Ma, R. M. Hill, J. L. Lowery, S. V. Fridrikh and G. C. Rutledge, Langmuir, 2005, 21, 5549-5554.

57. X. Tian, L. Yi, X. Meng, K. Xu, T. Jiang and D. Lai, Appl. Surf. Sci., 2014, 307, 566-575.

58. L. Yi, X. Meng, X. Tian, W. Zhou and R. Chen, J. Phts. Chem. C., 2014, 118, 26671-26682.

59. A. Alli, B. Hazer, Y. Menceloglu and E. Suzer, Eur. Polym. J., 2006, 42, 740-750.

60. R. Vasita, G. Mani, C. M. Agrawal and D. S. Katti, Polymer, 2010, 51, 3706-3714.

61. R. S. Kurusu and N. R. Demarquette, Langmuir, 2015, 31, 5495-5503.

62. R. S. Kurusu and N. R. Demarquette, Eur. Polym. J., 2017, 89, 129-137.

63. N. Detta, A. A. El Fattah, E. Chiellini, P. Walkenstrom and P. Gatenholm, J. Appl. Polym. Sci., 2008, 110, 253-261.

64. D. J. Yang, L. F. Zhang, L. Xu, C. D. Xiong, J. Ding and Y. Z. Wang, J. Biomed. Mater. Res. A, 2007, 82A, 680-688. 
65. S. B. Jiang and G. Y. Liao, Polym. Plast.Technol, 2012, 51, 1237-1244.

66. P. Viswanathan, E. Themistou, K. Ngamkham, G. C. Reilly, S. P. Armes and G. Battaglia, Biomacromolecules, 2015, 16, 66-75.

67. A. Nykänen, S. P. Hirvonen, H. Tenhu, R. Mezzenga and J. Ruokolainen, Polym. Int., 2014, 63, 37-43.

68. J. Li, C. Peng, Z. Wang and J. Ren, RSC Advances, 2018, 8, 17551-17557.

69. J. J. Li, L. T. Zhu and Z.-H. Luo, Chem. Eng. J., 2016, 287, 474-481.

70. J. J. Li, Y. N. Zhou, Z. D. Jiang and Z. H. Luo, Langmuir, 2016, 32, 13358-13366.

71. J. J. Li, Y. N. Zhou and Z. H. Luo, ACS Appl, Mater, Inter., 2015, 7, 19643-19650.

72. L. Wang, P. D. Topham, O. O. Mykhaylyk, J. R. Howse, W. Bras, R. A. L. Jones and A. J. Ryan, Adv. Mater., 2007, 19, 3544-3548.

73. J. You, W. Sheng, K. Huang, C. Hou, H. Yue, B. Hu, M. Wang, D. Wei, Q. Li, L. Zhao, W. Dong, Z. Zhao and Y. Li, ACS Appl. Mater. Inter., 2013, 5, 2278-2282.

74. W. Sheng, J. Zhao, Z. Chen, Q. Ye, X. Yang, K. Huang, C. Hou, J. You and Y. Li, Ind. Eng. Chem. Res., 2016, 55, 8292-8298.

75. S. M. Bhaway, Y. M. Chen, Y. Guo, P. Tangvijitsakul, M. D. Soucek, M. Cakmak, Y. Zhu and B. D. Vogt, Acs Appl. Mater. Inter., 2016, 8, 19484-19493.

76. M. M. Teng, J. L. Qiao, F. T. Li and P. K. Bera, Carbon, 2012, 50, 2877-2886.

77. R. Ravichandran, S. Gandhi, D. Sundaramurthi, S. Sethuraman and U. M. Krishnan, J. Biomat. Sci-Polym E., 2013, 24, 1988-2005.

78. R. Ravichandran, D. Sundaramurthi, S. Gandhi, S. Sethuraman and U. M. Krishnan, Micropor. Mesopor. Mat., 2014, 187, 53-62.

79. Y. Y. Zhao, H. Y. Wang, X. F. Lu, X. Li, Y. Yang and C. Wang, Mater. Lett., 2008, 62, 143-146.

80. V. Kalra, J. Lee, J. H. Lee, S. G. Lee, M. Marquez, U. Wiesner and Y. L. Joo, Small, 2008, 4, 2067-2073.

81. V. Kalra, J. H. Lee, J. H. Park, M. Marquez and Y. L. Joo, Small, 2009, 5, 2323-2332.

82. S. Q. Feng, X. Y. Shen and Y. L. Ji, J. Macromol. Sci. B., 2011, 50, 1673-1681.

83. X. D. Xu, J. C. Liu, G. K. Sheng, R. H. Chen, J. Zhao, X. J. Liu, Q. Shi and J. H. 
Yin, Appl. Surf. Sci., 2018, 451, 76-85.

84. J. Gao, H. Wang, X. Huang, M. Hu, H. Xue and R. K. Y. Li, J. Mater. Chem. A., 2018, 6, 10036-10047.

85. L. Wang, P. D. Topham, O. O. Mykhaylyk, H. Yu, A. J. Ryan, J. P. Fairclough, W. Bras, Macromol. Rapid Comm., 2015, 36, 1437-1443.

86. J. Wu, X. Li, Y. Wu, G. Liao, P. Johnston, P. D. Topham and L. Wang, Appl. Surf. Sci., 2017, 422, 769-777.

87. S. Q. Feng and X. Y. Shen, E-Polymers, 2010, -.

88. K. Kim, Y. K. Luu, C. Chang, D. F. Fang, B. S. Hsiao, B. Chu and M. Hadjiargyrou, J. Control. Release., 2004, 98, 47-56.

89. E. Gonzalez, L. M. Shepherd, L. Saunders and M. W. Frey, Materials, 2016, 9, 11 .

90. L. M. Shepherd, E. Gonzalez, E. X. Chen and M. W. Frey, ACS Appl Mater Inter, 2017, 9, 1968-1974.

91. A. K. Gaharwar, S. M. Mihaila, A. A. Kulkarni, A. Patel, A. Di Luca, R. L. Reis, M. E. Gomes, C. van Blitterswijk, L. Moroni and A. Khademhosseini, J. Control. Release., 2014, 187, 66-73.

92. L. Moroni, R. Licht, J. de Boer, J. R. de Wijn and C. A. van Blitterswijk, Biomaterials, 2006, 27, 4911-4922.

93. A. A. Deschamps, D. W. Grijpma and J. Feijen, Polymer, 2001, 42, 9335-9345.

94. H. Zhang, J. Xia, X. Pang, M. Zhao, B. Wang, L. Yang, H. Wan, J. Wu and S. Fu, Mat. Sci. Eng. C., 2017, 73, 537-543.

95. S. Z. Fu, X. H. Meng, J. Fan, L. L. Yang, Q. L. Wen, S. J. Ye, S. Lin, B. Q. Wang, L. L. Chen, J. B. Wu, Y. Chen, J. M. Fan and Z. Li, J. Biomed. Mater. Res. B., $2014, \mathbf{1 0 2}, 533-542$.

96. G. Guo, S. Z. Fu, L. X. Zhou, H. Liang, M. Fan, F. Luo, Z. Y. Qian and Y. Q. Wei, Nanoscale, 2011, 3, 3825-3832.

97. S. Z. Fu, X. H. Wang, G. Guo, S. A. Shi, H. Liang, F. Luo, Y. Q. Wei and Z. Y. Qian, J. Phys. Chem. C., 2010, 114, 18372-18378.

98. S. Z. Fu, P. Y. Ni, B. Y. Wang, B. Y. Chu, J. R. Peng, L. Zheng, X. Zhao, F. Luo, Y. 
Q. Wei and Z. Y. Qian, Biomaterials, 2012, 33, 8363-8371.

99. S. Z. Fu, L. L. Yang, J. Fan, Q. L. Wen, S. Lin, B. Q. Wang, L. L. Chen, X. H. Meng, Y. Chen and J. B. Wu, Colloid. Surface. B., 2013, 107, 167-173.

100. D. Vaikkath, R. Anitha, B. Sumathy and P. D. Nair, C Colloid. Surface. B., 2016, 141, 112-119.

101. F. Croisier, G. Atanasova, Y. Poumay and C. Jérôme, Adv. Healthc. Mater., 2014, 3, 2032-2039.

102. S. J. Cho, S. M. Jung, M. Kang, H. S. Shin and J. H. Youk, Polymer, 2015, 69, 95-102.

103. J. F. Pan, N. H. Liu, H. Sun and F. Xu, PLoS One, 2014, 9, 12.

104. P. U. Kadakia, E. A. G. Kalaf, A. J. Dunn, L. P. Shornick and S. A. Sell, J. Bioact. Compat. Polym., 2018, 33, 79-94.

105. J. Y. Gu, N. H. Liu, X. R. Yang, Z. H. Feng and F. Z. Qi, Biomed. Mater., 2014, 9, $35012-35021$.

106. G. Birhanu, H. A. Javar, E. Seyedjafari, A. Zandi-Karimi and M. D. Telgerd, Artif. Cell. Nanomed. B., 2018, 46, 1274-1281.

107. W. C. Li, Y. Q. Hu, L. Shi, X. L. Zhang, L. M. Xiong, W. C. Zhang and I. Ullah, J. Biomat. Sci-Polym E., 2018, 29, 1155-1167.

108. S. S. Zargarian and V. Haddadi-Asl, Artif. Cell. Nanomed. B., 2017, 45, 871-880.

109. S. S. Zargarian and V. Haddadi-Asl, Int. J. Polym. Anal. Charact., 2016, 21, 636-646.

110. H. T. Li, P. Song, T. K. Qiao, Q. Q. Cui, X. F. Song and B. C. Zhang, Polym. Adv. Technol., 2016, 27, 178-184.

111. A. Kyziol, J. Michna, I. Moreno, E. Gamez and S. Irusta, Eur. Polym. J., 2017, 96, $350-360$.

112. Y. L. Hong, H. S. Fan and X. D. Zhang, J. Phys. Chem. B., 2009, 113, 5837-5842.

113. A. Bhattacharjee, K. Kumar, A. Arora and D. S. Katti, Mat. Sci. Eng. C. Mater., 2016, 63, 266-273.

114. Q. Qi, T. Zhang, L. Liu, X. J. Zheng and G. Y. Lu, Sensor. Actuat. B. Chem., 2009, 141, 174-178. 
115. S. X. Chen, L. P. Ge, A. Mueller, M. A. Carlson, M. J. Teusink, F. D. Shuler and J. W. Xie, Nanomed. Nanotechnol., 2017, 13, 1435-1445.

116. K. Vocetkova, M. Buzgo, V. Sovkova, M. Rampichova, A. Staffa, E. Filova, V. Lukasova, M. Doupnik, F. Fiori and E. Amler, Rsc Advances., 2017, 7, $53706-53719$.

117. S. Xu, J. Y. Xia, S. J. Ye, M. Zhao, B. Q. Wang, L. L. Yang, J. B. Wu and S. Z. Fu, J. Appl. Polym. Sci., 2016, 133, 8, 1717-1726.

118. D. J. Yang, C. D. Xiong, T. Govender and Y. Z. Wang, J. Biomater. Sci.-Polym. Ed., 2009, 20, 1321-1334.

119. Q. Li, Z. H. Wang, Q. Zhao, L. Y. Wang, S. F. Wang and D. L. Kong, E-Polymers., 2012

120. Y. Ner, J. A. Stuart, G. Whited and G. A. Sotzing, Polymer., 2009, 50, 5828-5836.

121. P. Tzeng, C. C. Kuo, S. T. Lin, Y. C. Chiu and W. C. Chen, Macromol. Chem. Phys., 2010, 211, 1408-1416.

122. Y. C. Chiu, Y. Chen, C. C. Kuo, S. H. Tung, T. Kakuchi and W. C. Chen, ACS Appl. Mater. Inter., 2012, 4, 3387-3395.

123. J. T. Wang, Y. C. Chiu, H. S. Sun, K. Yoshida, Y. Chen, T. Satoh, T. Kakuchi and W. C. Chen, Polym. Chem., 2015, 6, 2327-2336.

124. A. Kapllani, C. Tran and V. Kalra, Soft Matter., 2013, 9, 11014-11020.

125. B. Grignard, A. Vaillant, J. de Coninck, M. Piens, A. M. Jonas, C. Detrembleur and C. Jerome, Langmuir., 2011, 27, 335-342.

126. S. J. Peng, G. R. Jin, L. L. Li, K. Li, M. Srinivasan, S. Ramakrishna and J. Chen, Chem. Soc. Rev., 2016, 45, 1225-1241.

127. R. J. Wade and J. A. Burdick, Mater. Today., 2012, 15, 454-459.

128. A. Jindal, J. E. Puskas, A. McClain, K. Nedic, M. T. Luebbers, J. R. Baker, B. P. dos Santo, M. Camassola, W. Jennings, R. L. Einsporn and N. D. Leipzig, Eur. Polym. J., 2018, 98, 254-261.

129. M. S. V. Ferreira and S. H. Mousavi, Nanomed. Nanotechnol., 2018, 14, $1707-1718$

130. A. K. Gaharwar, S. M. Mihaila, A. A. Kulkarni, A. Patel, A. Di Luca, R. L. Reis, 
M. E. Gomes, C. van Blitterswijk, L. Moroni and A. Khademhosseini, J. Control. Release., 2014, 187, 66-73.

131. C. Mota, S. Danti, D. D'Alessandro, L. Trombi, C. Ricci, D. Puppi, D. Dinucci, M. Milazzo, C. Stefanini, F. Chiellini, L. Moroni and S. Berrettini, Biofabrication., $2015,7,025005$.

132. S. Demirci, A. Celebioglu, Z. Aytac and T. Uyar, Polym. Chem., 2014, 5, 2050-2056.

133. E. Gonzalez, L. M. Shepherd, L. Saunders and M. W. Frey, Materials, 2016, 9, 47-58.

134. A. J. Keefe, N. D. Brault and S. Jiang, Biomacromolecules, 2012, 13, 1683-1687.

135. P. Patel, C. K. Choi and D. D. Meng, JALA-J. Lab. Autom., 2010, 15, 114-119.

136. J. F. Pan, N. H. Liu, L. Y. Shu and H. Sun, J. Nanobiotechnol., 2015, 13, 37-51.

137. W. Mao and H. S. Yoo, Macromol. Biosci., 2017, 17, 10, 1700057.

138. A. Valizadeh, M. Bakhtiary, A. Akbarzadeh, R. Salehi, S. M. Frakhani, O. Ebrahimi, M. Rahmati yamchi and S. Davaran, Artif. Cell. Nanomed. Biotechnol., $2016,44,504509$.

13839. R. Machado, A. da Costa, V. Sencadas, C. Garcia-Arevalo, C. M. Costa, J. Padrao, A. Gomes, S. Lanceros-Mendez, J. C. Rodriguez-Cabello and M. Casal, Biomed. Mater., 2013, 8, 065009.

13940. W. Qiu, J. Cappello and X. Wu, Appl. Phys. Lett., 2011, 98, 3702-37023.

14041. W. Qiu, Y. Huang, W. Teng, C. M. Cohn, J. Cappello and X. Wu, Biomacromolecules., 2010, 11, 3219-3227.

141z.J. M. Nunzi, C. R. Phys., 2002, 3, 523-542. 